\title{
A TAXONOMIC REVISION OF CANSCORA, CRACOSNA, DUPLIPETALA, HOPPEA, MICRORPHIUM, PHYLLOCYCLUS AND SCHINZIELLA (GENTIANACEAE-CANSCORINAE)
}

\author{
MIKE THIV \\ Institute of Systematic Botany, University of Zurich, Zollikerstr. 107, \\ CH-8008 Zurich, Switzerland; e-mail: thiv@ systbot.unizh.ch
}

SUMMARY

A taxonomic revision of all genera belonging to Gentianaceae-Canscorinae, i.e., Canscora, Cracosna, Duplipetala, Hoppea, Microrphium, Phyllocyclus and Schinziella is presented, including keys to the taxa, distribution maps and seed coat descriptions. One new genus, one new species and seven new combinations are described. In total seven genera and 23 species are recognized. All taxa are of palaeotropical distribution with centres in India and SE Asia.

Key words: Gentianaceae, Canscora, Cracosna, Duplipetala, Hoppea, Microrphium, Phyllocyclus, Schinziella, taxonomy.

\section{INTRODUCTION}

A molecular phylogenetic analysis of the Gentianaceae based on the plastid matK, $\operatorname{tr} n \mathrm{~L}$-intron and partial $r b c \mathrm{~L}$ sequence data was the basis for a new classification of the family with six tribes and eight subtribes (Thiv et al., 1999a, b; Thiv, 2000; Struwe et al., 2002). On the basis of these studies, subtribe Canscorinae Thiv \& Kadereit as part of tribe Chironieae (G. Don) Endl. was established (Struwe et al., 2002). The Canscorinae are characterized by their palaeotropical distribution, a calyx tube that is much longer than the lobes, tetra-, penta- or hexamerous flowers, and often anisomorphic stamens. The subtribe contains 23 species in seven genera. These are Canscora Lam., Cracosna Gagnep., Duplipetala Thiv, Hoppea Willd., Microrphium C.B. Clarke, Phyllocyclus Kurz and Schinziella Gilg.

Most authors in the early 19th century treated Canscora and Hoppea as members of the same tribe or subtribe (Don, 1838; Endlicher, 1838; Grisebach, 1839, 1845; Table 1). Gilg (1895) regarded the genera of Canscorinae known at that time as part of his Gentianeae-Erythraeinae together with several genera, which do not belong to this subtribe in the molecular analyses. Gilg-Benedict (1939) placed Canscora, Hoppea, and Schinziella in the Gentianeae-Erythraeinae-Ellipandrae, but ignored Microrphium, Phyllocyclus, and Cracosna although Gagnepain (1929) suspected a close relationship between the latter genus and Canscora.

Canscora was described by Lamarck (1785) based on $C$. perfoliata. Willdenow (1801) described Hoppea with H.dichotoma, and Kurz (1873) established Phyllocyclus, which was later reduced to subgeneric rank in the classification of Canscora by Clarke (1885). Clarke divided Canscora into subgenera Canscora Lam., Heterocanscora 


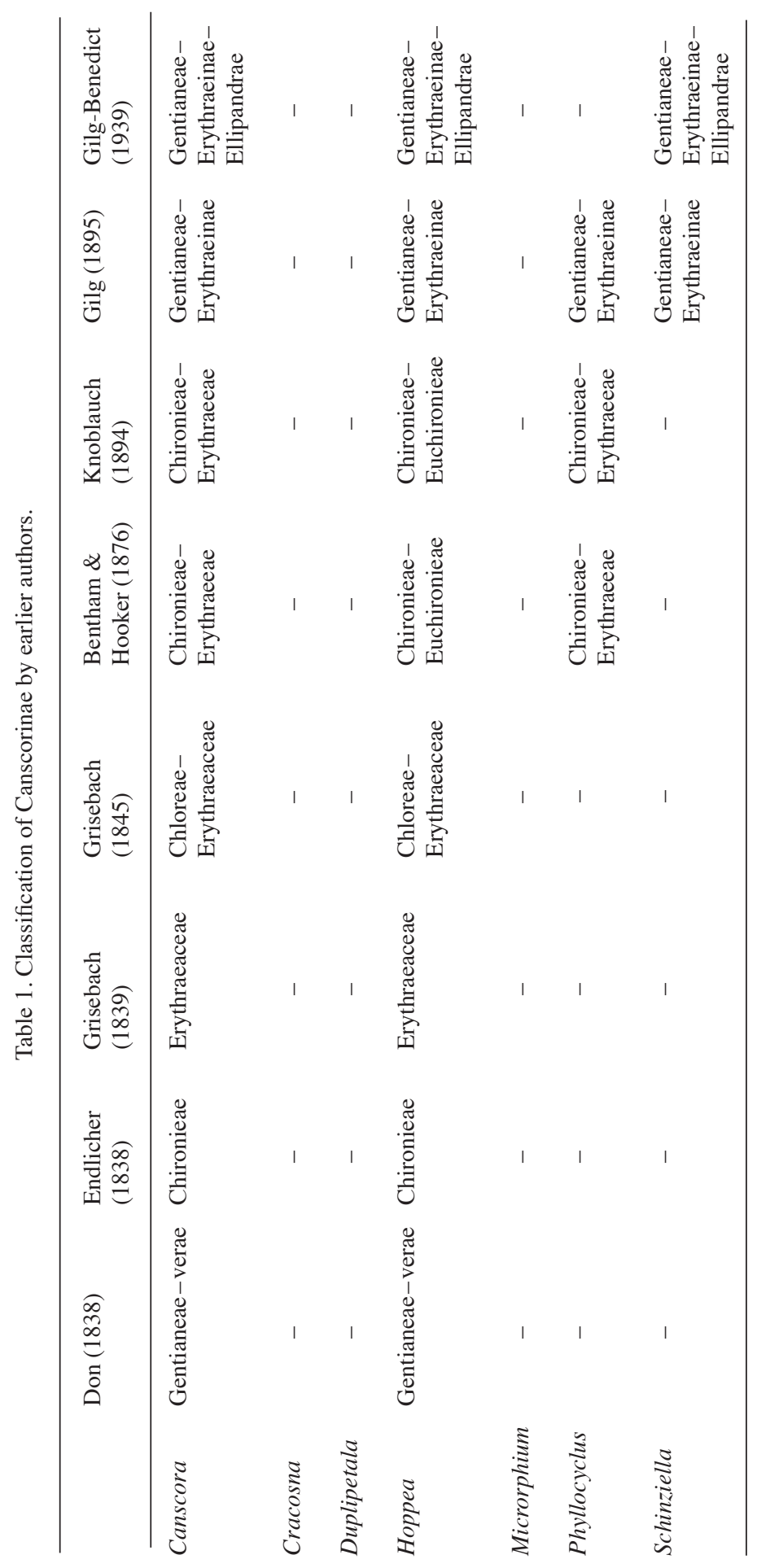


Table 2. Classification of Canscora by Clarke $(1885,1906)$.

\begin{tabular}{ll}
\hline Subgenus & Species \\
\hline Canscora & C. diffusa \\
& C. heteroclita \\
& C. alata \\
& C. concanensis \\
& C.perfoliata \\
& C. roxbughii \\
& C. andrographioides \\
& C. schultesii \\
Heterocanscora & C.helferiana \\
Phyllocyclus & C.parishii \\
& C.pentanthera \\
\hline
\end{tabular}

(C.B. Clarke) C.B. Clarke, and Phyllocyclus (Kurz) C.B. Clarke. In 1895 Gilg transferred Canscora tetragona to a new genus, Schinziella. Clarke (1906) not only described the new genus Microrphium, but also the new subgenus Pentanthera C.B. Clarke of Canscora. The new genus Cracosna was established by Gagnepain (1929), its name being an anagram of Canscora. The majority of the 23 species of the Canscorinae were originally described as species of Canscora. Previously, most authors (e.g., Ridley, 1923; Ubolcholaket, 1987; Ho \& Pringle, 1995) accepted Clarke's $(1885,1906)$ treatment of Canscora as a large genus comprising four subgenera (Table 2).

Because the taxonomic treatment of the Canscorinae by earlier authors was very incomplete, the generic and specific delimitations remained hitherto unclear. Therefore, the following revision provides a complete overview of the subtribe. In this paper a narrower definition of Canscora is preferred, and a new genus, Duplipetala, is described. The combination of molecular and morphological evidence (Thiv et al., 1999b; Thiv \& Kadereit, 2002) leads to the recognition of three major lineages within the Canscorinae: 1. Canscora s.str. (= subgenera Canscora and Heterocanscora), Hoppea, and Schinziella have tetramerous flowers and an anisomorphic androecium; 2. although only weakly supported, Cracosna, which is characterized by the possession of tetramerous flowers, isomorphic stamens and petal nectaries, was found to be the sister group to Canscora s.str. / Hoppea / Schinziella (Thiv \& Kadereit, 2002); 3. Microrphium, Phyllocyclus (= Canscora subgenus Phyllocyclus), and Duplipetala (= Canscora subgenus Pentanthera), have penta- or hexamerous flowers and an isomorphic androecium in which, however, the length of the filaments can differ.

\section{MATERIAL AND METHODS}

For the morphological studies herbarium material from A, B, BKF, BM, BO, BR, C, E, F, FWM, G, HBG, K, KLU, KUN, L, MEL, MICH, MO, NSW, NY, P, PE, PH, PNH, S, SING, SRGH, TUB, U, US and Z was used. Distribution maps were created using Online Map Creation (http://www.aquarius.geomar.de/omc/) and modified in Adobe Illustrator 9.0. For the SEM-photography seeds were stuck on aluminium stubs with double-sided tape and sputter-coated with gold on a Balzers SCD 004 sputter. SEMphotographs were taken on a LEITZ AMR 1200 B microscope. 


\section{TAXONOMIC TREATMENT}

CANSCORINAE Thiv \& Kadereit in Struwe et al. (2002) 50

Annual or perennial, rarely suffrutescent, mostly erect and glabrous herbs; stems quadrangular or rarely terete, unwinged or often strongly winged. Rosette leaves present or not; cauline leaves free or rarely perfoliate, sessile or rarely petiolate. Inflorescences axillary or rarely terminal cymes or rarely spikes; bracts free or perfoliate. Flowers tetra-, penta-, or hexamerous (sometimes the number of calyx lobes reduced), sessile or pedicellate, with or without bracteoles. Calyx tubular, funnelshaped, campanulate or inflated urceolate, sometimes strongly winged, mostly persistent; tube much longer than lobes; calycine colleters absent. Corolla actinomorphic or zygomorphic (see Canscora), tubular, salver- or funnelshaped; tube mostly longer than lobes. Androecium isomorphic or anisomorphic (see genus descriptions), inserted in upper, central or lower part of corolla tube; anthers basifixed, introrse, persistent or deciduous, mostly sagittate; pollen in monads. Ovary of two carpels, unilocular; placentation parietal; style distinct, filiform; stigma bilobed. Fruit a septicidal capsule with numerous seeds. Seeds of various shape.

\section{KEY TO THE GENERA OF CANSCORINAE}

1a. 1 (or rarely 2) stamen inserted at higher level than the remaining stamens, this stamen mostly with larger anther; anthers persistent. Flowers tetramerous. Mainly annuals, when perennials, flowers in dense, head-like cymes. Cauline leaves free

b. All stamens inserted at the same level, anthers of equal size; caducous (except for Cracosna). Flowers (corolla and androecium) tetra-, penta-, or hexamerous. Annuals or perennials with flowers in lax or dense, never headlike cymes or spikes. Cauline leaves free or perfoliate $\ldots \ldots \ldots \ldots \ldots \ldots \ldots \ldots \ldots \ldots \ldots \ldots \ldots \ldots \ldots \ldots \ldots$

2a. Plants perennial, often suffrutescent. Corolla yellow, actinomorphic. Flowers in

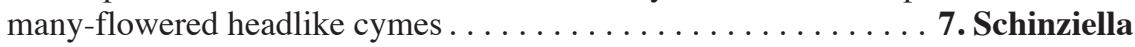

b. Plants annual, herbaceous. Corolla whitish or lilac, actinomorphic or zygomorphic. Flowers in lax or dense cymes or rarely spikes . . . . . . . . . . . 3

3a. Corolla $<4.4 \mathrm{~mm}$, actinomorphic, white. Stems with wings . . . . 4. Hoppea

b. Corolla $>5.2 \mathrm{~mm}$, zygomorphic, with 2 larger lobes and 2 smaller lobes, whitish

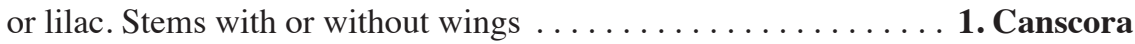

4a. Plants with bristles on green parts (peduncles, calyx base and young leaves). Calyx

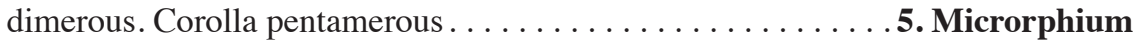

b. Plants entirely glabrous. Never a combination of a dimerous calyx and a penta-

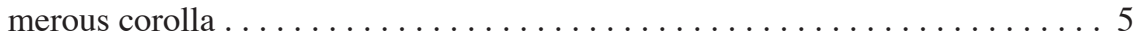

5a. Corolla tetramerous with nectaries in the middle of the inner surface ${ }^{1}$. Calyx tubular. Flowers in dense or lax cymes. All leaves including bracts free. Stems winged . .

2. Cracosna

b. Corolla penta- or hexamerous without nectaries. Calyx mostly urn-shaped. Flowers in lax cymes. Bracts and/or cauline leaves perfoliate or free. Stems not winged. 6

1) Nectaries are sometimes difficult to observe in herbarium material. 
6a. Cauline leaves free. Filaments usually not broadened at the base. Calyx venation reticulate. Bracts free or perfoliate, apex of bracts acute. Corolla mostly hexa-

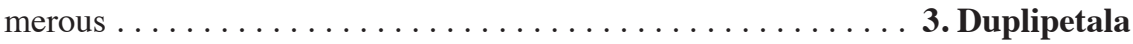

b. Cauline leaves perfoliate. Filaments broadened at the base. Calyx venation not reticulate. Bracts perfoliate, orbiculate; apex of bracts obtuse. Corolla mostly

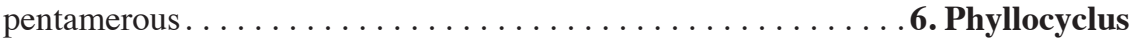

Notes - The main axis of Canscorinae shows monopodial branching. Cymes are defined as flower-bearing branches of the first order.

Whether 2- or 3-lobed calyces (e.g. in Microrphium, Cracosna, Duplipetala) represent flower merosity or are the product of sepal-fusion still needs to be investigated.

\section{CANSCORA Lam.}

Canscora Lam. (1785) 601. - Type: Canscora perfoliata Lam.

Orthostemon R.Br. (1810) 451. - Type: Orthostemon erectus R.Br.

Pladera Sol. ex Roxb. (1820) 416 p.p. - Type: Pladera sessiliflora Roxb.

Heterocanscora C.B. Clarke (1875) 431. - Type: Heterocanscora schultesii (Wall. ex Griseb.)

C.B. Clarke.

Annual, erect or rarely creeping, glabrous herbs; stems quadrangular, often strongly winged. Rosette leaves rarely present; cauline leaves free, sessile or rarely petiolate, lamina with one or three main veins. Inflorescences axillary cymes or rarely spikes; bracts free or perfoliate. Flowers tetramerous, sessile or rarely pedicellate, without bracteoles. Calyx tubular, sometimes strongly winged, mostly persistent or rarely disintegrating; lobes triangular to lanceolate. Corolla zygomorphic with two smaller and two larger lobes (Fig. 2c), salver- to funnelshaped, mostly white or pinkish purple; tube longer than lobes. Androecium anisomorphic (Fig. 2c), inserted in upper part of corolla tube; stamen between the two smaller corolla lobes inserted at higher level (upper stamen) than the remaining stamens (lower stamens), often with larger anther and longer filament which sometimes is broadened below the anther; anthers sagittate, persistent. Ovary oblong; stigmatic lobes oblong. Fruit an oblong capsule. Seeds irregular in shape, subglobose or angular, often cubical to rectangular and with shallowly sunken sides; outer testa reticulate, with shallow cells; testa cells irregularly polygonal, oriented irregularly; anticlinal walls prominent, mostly curved or straight; cuticle smooth.

Distribution - Nine species in tropical Asia, Africa and Australia.

\section{KEY TO THE SPECIES OF CANSCORA}

1a. Flowers arranged in a spike. Rosette leaves present . . . . . 1.9. C. schultesii

b. Flowers arranged in lax cymes. Rosette leaves absent . . . . . . . . . . 2

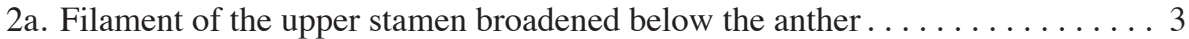

b. Filaments of the upper stamen not broadened below the anther . . . . . . . 6

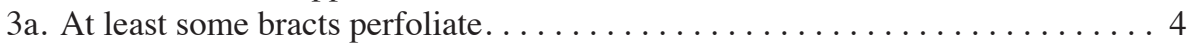

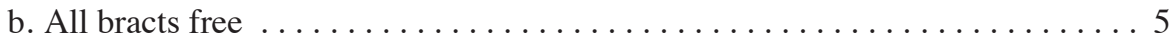

4a. Corolla 13-14 mm long. Calyx with wings up to $3.5 \mathrm{~mm}$ wide 1.7. C. perfoliata

b. Corolla 9-10 mm long. Calyx without wings .......... 1.8. C. roxburghii 

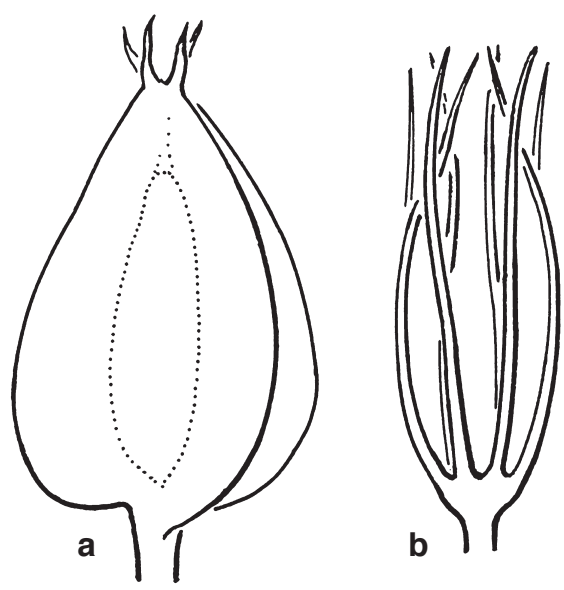

Fig. 1. a. Calyx of Canscora macrocalyx Miq.; b. disintegrating calyx at fruit maturity of $C$. andrographioides Griff. ex C.B. Clarke.

5a. Calyx with wings up to $3.5 \mathrm{~mm}$ wide, wings largest in lower part of calyx (Fig. 1).

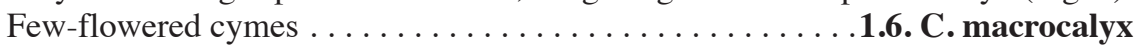

b. Calyx with 1-1.3 mm wide wings, wings \pm equally wide along the calyx. Many-

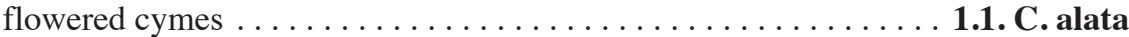

6a. Calyx with wings $1-1.6 \mathrm{~mm}$ wide $\ldots \ldots \ldots \ldots \ldots \ldots$ 1.3. C. concanensis

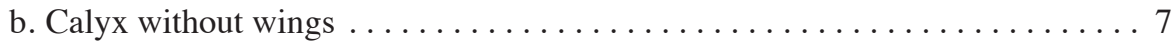

7a. Flowers sessile. Leaves $<15 \mathrm{~mm}$ long, ovate. Stems strongly winged . . . . . . .

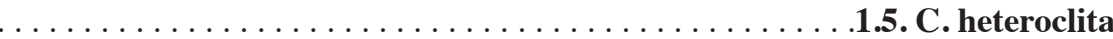

b. At least some flowers pedicellate. Leaves $>20 \mathrm{~mm}$ long, of various shape. Stems

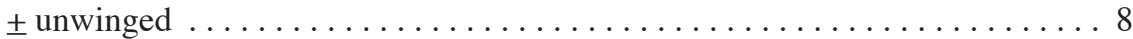

8a. Calyx $>10 \mathrm{~mm}$ long, 3.5-7 times longer than wide, sometimes disintegrating at fruit maturity (Fig. 1). Corolla $>15 \mathrm{~mm}$ long. Anthers of different size. Flowers in 1-3-flowered axillary cymes. Leaves sometimes falcate

1.2. C. andrographioides

b. Calyx $<7 \mathrm{~mm}$ long, 1.5-5 times longer than wide, persistent at fruit maturity. Corolla $<14 \mathrm{~mm}$ long. Anthers \pm of equal size. Flowers in 3-many-flowered cymes. Leaves never falcate

1.4. C. diffusa

Note - In contrast to Cracosna which mostly grows in dry habitats, Canscora, Hoppea and Schinziella prefer moist places. The occurrence of many taxa like Canscora diffusa and $C$. alata along rivers may indicate seed dispersal by water.

\subsection{Canscora alata (Roth) Wall. - Plate $1 \mathrm{~g}$, h; Map 1,2}

Canscora alata (Roth) Wall. (1831) 4363. - Exacum alatum Roth (1818) 159. - Type: Hildebrandt 3303 (neo P, fide Klackenberg (1990); iso BM, K n.v.), Madagascar, Statio Nosi-Be, Dec. 1879.

Pladera decussata Roxb. (1820) 418. - Canscora decussata (Roxb.) Roem. \& Schult. (1827) 229. - Type: Roxburgh Ic. No. 236 (K n.v.).

Plants 5-55 cm tall; stems basally and apically branched, with prominent wings up to $1.6 \mathrm{~mm}$ wide. Cauline leaves sessile, lamina with three main veins, elliptic to lanceolate, often falcate, $11-38$ by $7-27 \mathrm{~mm}$, base wedge-shaped to shortly attenuate, apex acute. Inflorescences axillary, 1-7-flowered cymes; bracts linear to lanceolate, 4.5-12 
by $1-4 \mathrm{~mm}$; pedicels $2-26 \mathrm{~mm}$ long. Calyx $11-14$ by $2-3 \mathrm{~mm}$, persistent, with eight prominent, $1-1.3 \mathrm{~mm}$ wide wings; tube $8-9 \mathrm{~mm}$ long; lobes anisomorphic, acute, two larger lobes 1.8 by $1.2 \mathrm{~mm}$ long, two shorter lobes 1.5 by $1.2 \mathrm{~mm}$. Corolla tube $8-9$ $\mathrm{mm}$ long, white, two lobes broadly elliptic, $4-4.5$ by $2-2.5 \mathrm{~mm}$, the other two lobes elliptic, 3 by $1.5 \mathrm{~mm}$. Filament of upper stamen $1.8-2.2 \mathrm{~mm}$ long, broadened below anther, anther $0.8-0.9$ by $0.6 \mathrm{~mm}$; filaments of lower stamens $1.5-2 \mathrm{~mm}$ long, anthers $1-1.1$ by $0.4-0.5 \mathrm{~mm}$. Ovary $6-8$ by $1.5-1.8 \mathrm{~mm}$; style up to $4 \mathrm{~mm}$ long; stigmatic lobes 0.7 by $0.4 \mathrm{~mm}$. Capsule 8 by $4 \mathrm{~mm}$. Seeds irregular in shape, cubical to rectangular and with shallowly sunken sides, c. 0.38 by $0.21 \mathrm{~mm}$; outer testa reticulate, with shallow cells; testa cells irregularly polygonal, oriented irregularly; anticlinal walls curved.

Distribution - Tropical Africa and S Asia.

Ecology - Shady, rocky river banks, grasslands, swamps, savannahs; sandy clayish soil. Sea level to $2500 \mathrm{~m}$ altitude. Flowering: throughout the year.

Chromosome number $-2 \mathrm{n}=76$ (Mallikarjuna et al., 1989).
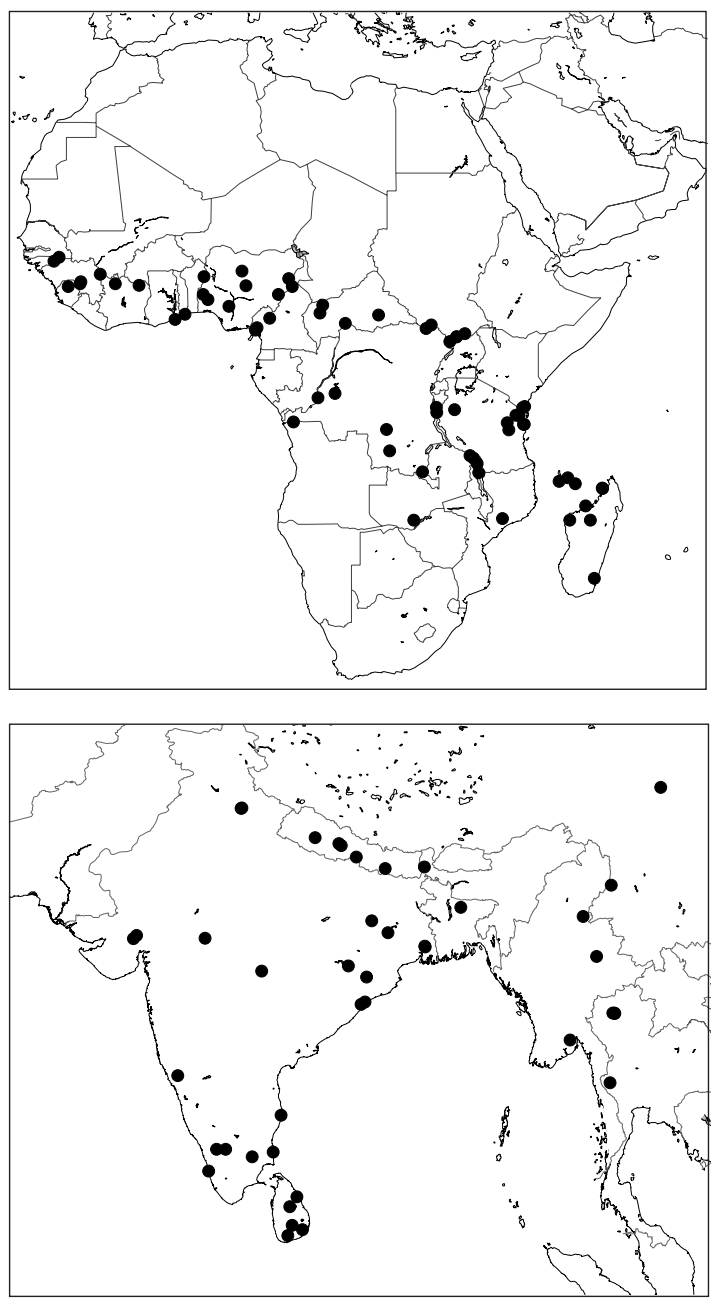

Map 1. Distribution of Canscora alata (Roth) Wall. in Africa.

Map 2. Distribution of Canscora alata (Roth) Wall. in Asia. 

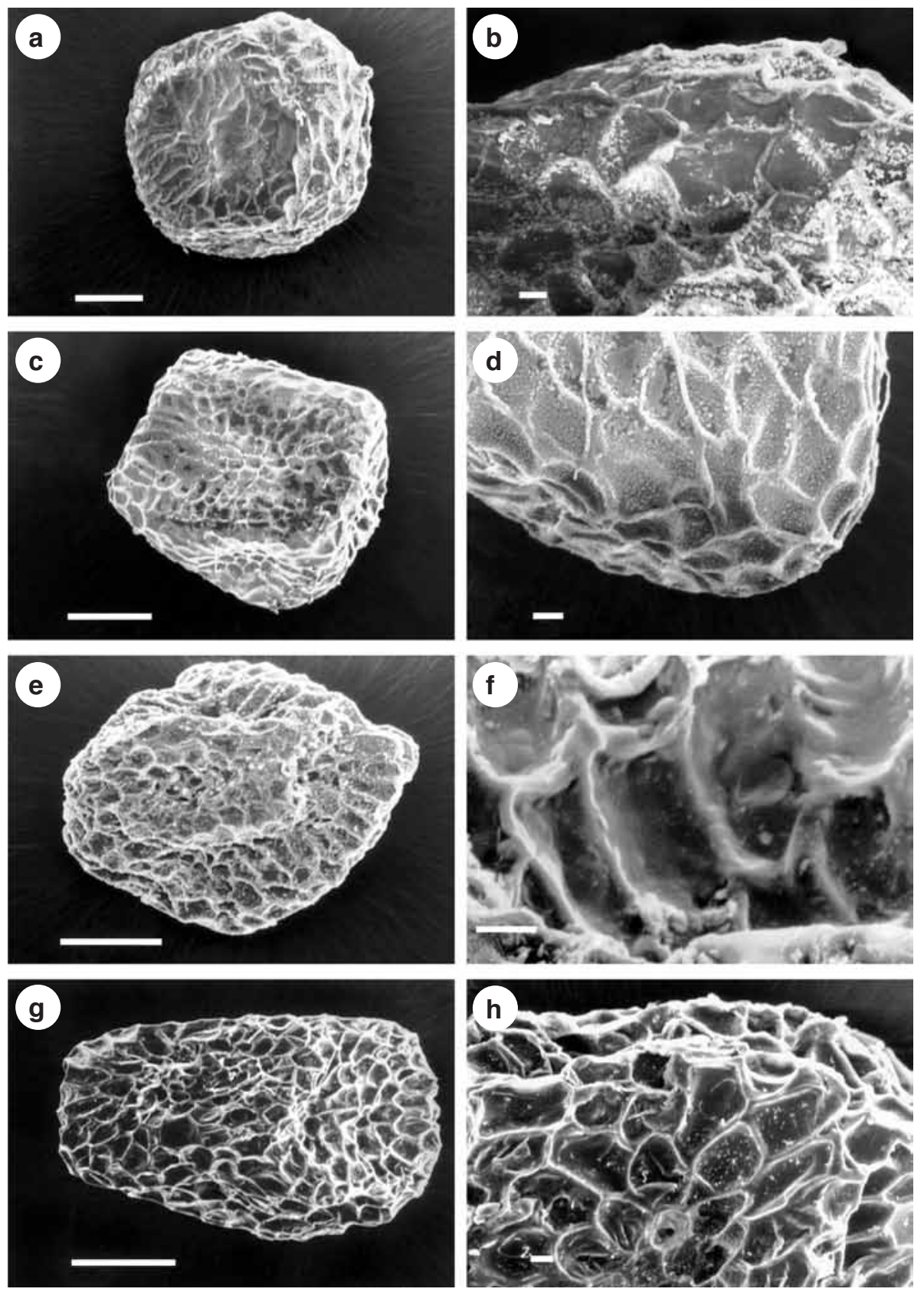

Plate 1. SEM photographs of seed coat structures. - a, b. Canscora perfoliata Lam. (Thompson s.n.); c, d. C. roxburghii Arn. ex Miq. (Cramer 4926); e, f. C. macrocalyx Miq. (Kooper s.n.); g, h. C. alata (Roth) Wall. (Wyld 324). - Scale bars: a, c, e, g $=100 \mu \mathrm{m} ; \mathrm{b}, \mathrm{d}, \mathrm{f}, \mathrm{h}=10 \mu \mathrm{m}$. 
1.2. Canscora andrographioides Griff. ex C.B. Clarke - Fig. 1; Plate 2a, b; Map 3

Canscora andrographioides Griff. ex C.B. Clarke (1875) 431. - Type: Griffith 5816/1 (lecto K, designated here; iso A), Khasya.

Canscora trinervia Ridl. (1908) 316. - Type: Ridley 5507 (lecto BM, designated here), Gunung Sakau, Pahang, 10 July 1905.

Canscora melastomacea Hand.-Mazz. (1932) 131. - Type: Ching 7868 (lecto A, designated here; iso NY), China, Kwangsi, S Nanning, Seh-Feng, Dar Shan, 1200 ft, 15 Oct. 1928.

Plants erect to creeping, 6-80 cm tall; stems basally and apically branched, with minute ridges. Cauline leaves petiolate, petiole 1-13 $\mathrm{mm}$ long, lamina with three main veins, lanceolate to elliptic, often slightly falcate, $20-60$ by 7-27 mm, base attenuate, apex acute. Inflorescences axillary, 1-3(-7)-flowered cymes; bracts linear to lanceolate, 2-20 by $0.5-7 \mathrm{~mm}$; pedicels absent or up to $21 \mathrm{~mm}$ long. Calyx $11-14$ by $2-3 \mathrm{~mm}$, sometimes disintegrating (Fig. 1), with 8 ridges; tube $9-11 \mathrm{~mm}$ long; lobes beaked to taper-pointed, 1.8 by $0.4 \mathrm{~mm}$. Corolla tube $11-16 \mathrm{~mm}$ long, white, two lobes broadly elliptic, $4.2-7.5$ by $2.8-3.5 \mathrm{~mm}$, the other two lobes elliptic, $3-4.5$ by $1.8-3 \mathrm{~mm}$. Filament of upper stamen $2-2.5 \mathrm{~mm}$ long, not broadened below anther, anther 1.3 by $1 \mathrm{~mm}$; filaments of lower stamens $0.4-1.1 \mathrm{~mm}$ long, anthers $0.7-1$ by $0.4-0.5 \mathrm{~mm}$. Ovary $5-9$ by $1-1.3 \mathrm{~mm}$; style up to $9 \mathrm{~mm}$ long; stigmatic lobes 0.6 by $0.5 \mathrm{~mm}$. Capsule $7-10$ by $2-3.5 \mathrm{~mm}$. Seeds irregular in shape, cubical to rectangular and with shallowly sunken sides, c. 0.50 by $0.38 \mathrm{~mm}$; outer testa reticulate, with shallow cells; testa cells irregularly polygonal, oriented irregularly; anticlinal walls straight.

Distribution - Tropical S and SE Asia.

Ecology - Understorey of dipterocarp or evergreen forests, roadsides, grassland, swamps, rocky banks of streams; open clay grounds. From sea level to $2300 \mathrm{~m}$ altitude. Flowering: throughout the year.

Note - This species is extremely variable in habit and branching. No morphological characters were found which distinguish this species from Canscora trinervia which was described from West Malaysia and purported to have a smaller size and thinner leaves.

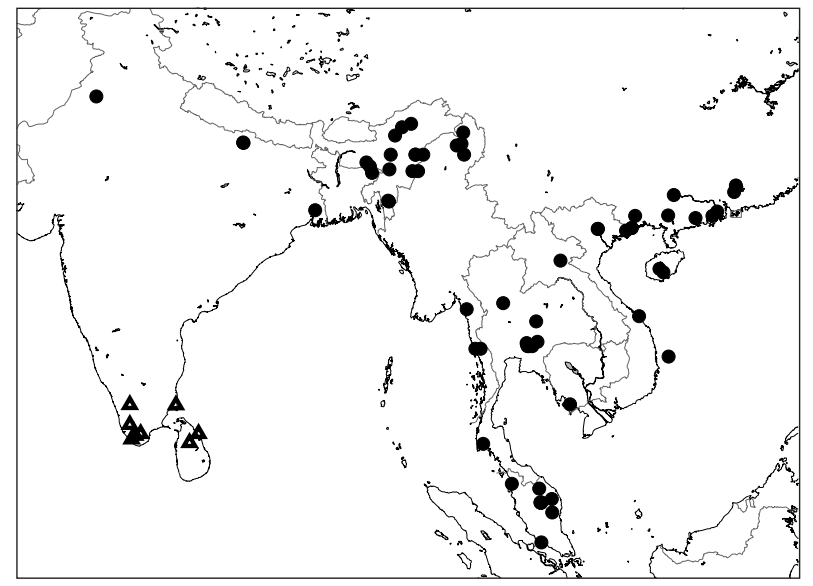

Map 3. Distribution of Canscora andrographioides Griff. ex C.B. Clarke (-) and C. roxburghii Arn. ex Miq. ( $\mathbf{\Delta})$. 

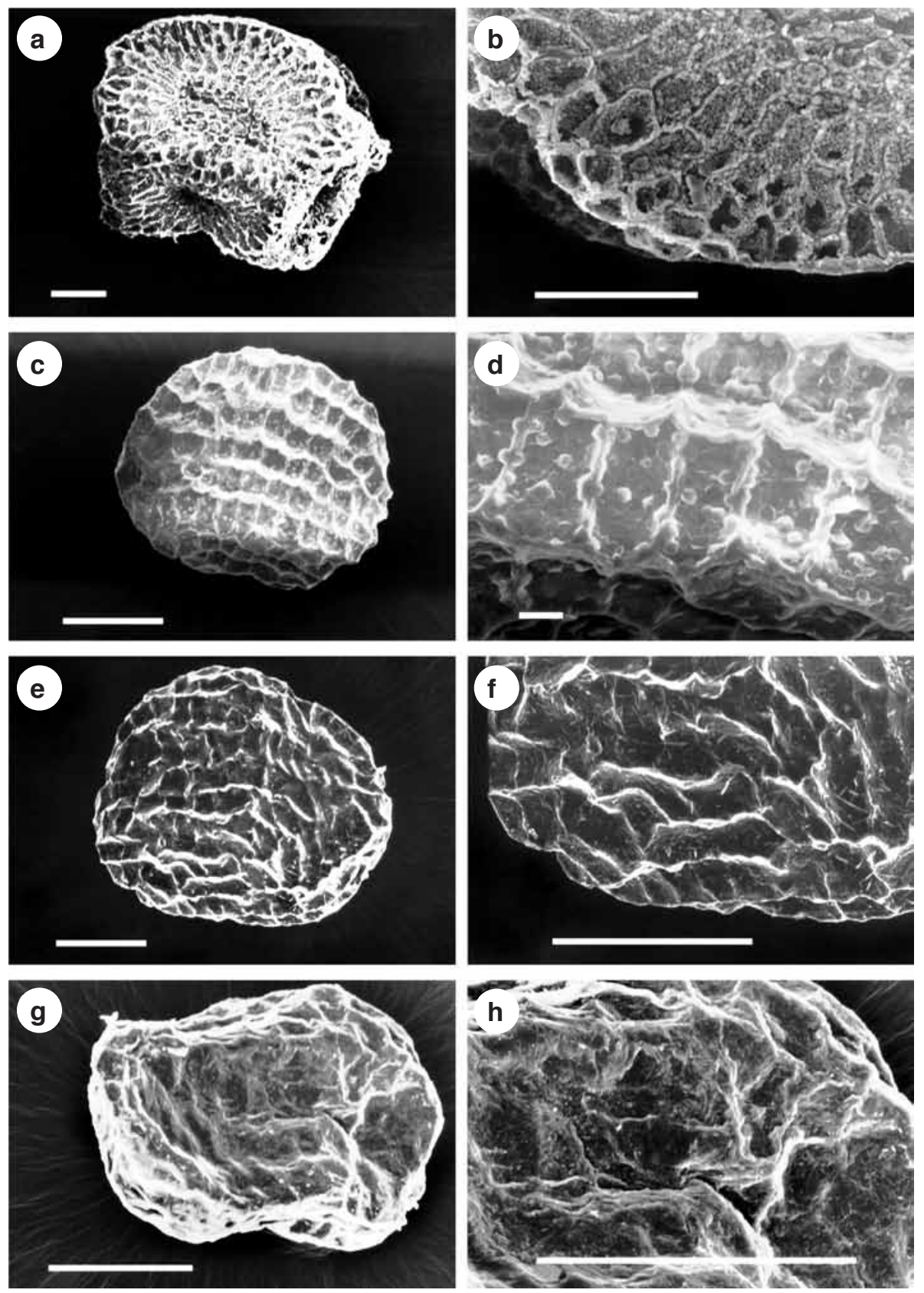

Plate 2. SEM photographs of seed coat structures. - a, b. Canscora andrographioides Griff. ex C.B. Clarke (Koelz 29638); c, d. C. concanensis C.B. Clarke (Radcliffe-Smith 5307); e, f. C. diffusa (Vahl) R.Br. ex Roem. \& Schult. (Koyama 15568); g, h. C. diffusa (Koelz 4599). - Scale bars: a-c, $\mathrm{e}-\mathrm{h}=100 \mu \mathrm{m} ; \mathrm{d}=10 \mu \mathrm{m}$. 
1.3. Canscora concanensis C.B. Clarke - Plate 2c, d; Map 4

Canscora concanensis C.B. Clarke (1885) 104. - Type: Law s.n. (holo K), India, Concoon.

Plants 8-26 cm tall; stems apically and partly basally branched, with minute ridges up to $0.1 \mathrm{~mm}$ wide. Cauline leaves sessile or rarely petiolate, petiole up to $6 \mathrm{~mm}$ long, lamina with one main vein, elliptic to lanceolate, $11-32$ by $5-16 \mathrm{~mm}$, base attenuate to shortly attenuate, apex acute. Inflorescences axillary, 1-6-flowered cymes; bracts linear to lanceolate, $1-6.8$ by $0.2-2.6 \mathrm{~mm}$; pedicels $3-15 \mathrm{~mm}$ long. Calyx $6-7.5$ by 3-5 mm, persistent, with 4 prominent wings $1-1.6 \mathrm{~mm}$ wide; tube 5-6 mm long; lobes triangular, acute, 1 by $1.2 \mathrm{~mm}$. Corolla tube $6-7 \mathrm{~mm}$ long, pink, two lobes broadly elliptic, $2.3-3$ by $1.5-2 \mathrm{~mm}$, the other two lobes elliptic, $1.3-1.5$ by $0.7-0.8 \mathrm{~mm}$. Filament of upper stamen $0.6-1 \mathrm{~mm}$ long, not broadened, anther $0.5-0.7$ by $0.3-0.4 \mathrm{~mm}$; filaments of lower stamens $0.7-1.1 \mathrm{~mm}$ long, anthers $0.6-0.8$ by $0.3-0.4 \mathrm{~mm}$. Ovary $3.5-5$ by $1.2-1.3 \mathrm{~mm}$; style $8-9 \mathrm{~mm}$ long; stigmatic lobes 0.4 by $0.3 \mathrm{~mm}$. Capsule $4-5$ by $1.5 \mathrm{~mm}$. Seeds subglobose to angular, c. 0.28 by $0.25 \mathrm{~mm}$; anticlinal walls straight; testa cells oriented in \pm linear rows.

Distribution - Oman, India.

Ecology - Open grasslands, roadsides or woodlands. Sea level to $700 \mathrm{~m}$ altitude. Flowering: September to November.

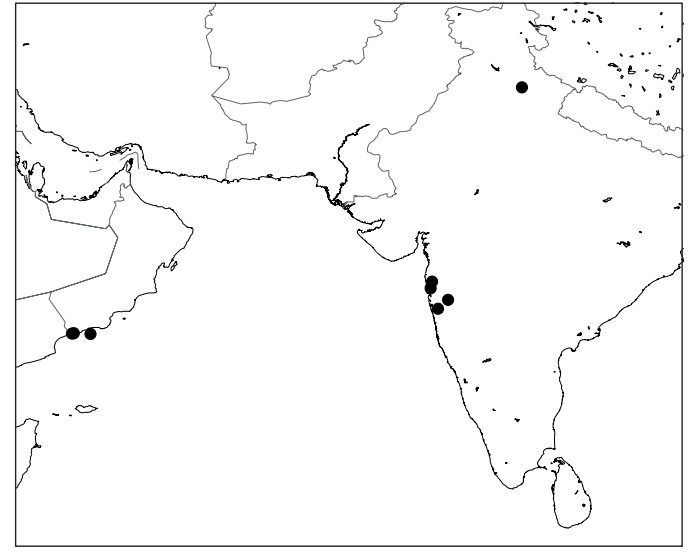

Map 4. Distribution of Canscora concanensis C.B. Clarke

1.4. Canscora diffusa (Vahl) R.Br. ex Roem. \& Schult. - Plate 2e-h; Map 5, 6

Canscora diffusa (Vahl) R.Br. ex Roem. \& Schult. (1818) 301. - Gentiana diffusa Vahl (1794) 47. - Exacum diffusum (Vahl) Willd. (1798) 637. - Type: Vahl s.n. (holo C), India orientalis.

Orthostemon erectus R.Br. (1810) 451. - Type: Banks \& Solander 1770 (holo BM), New Holland, Endeavour River.

Canscora tenella Wall. (1831) 4362, nom. nud.

Canscora decurrens Dalzell (1850) 136. - Type: Anonymous s.n. (K).

Canscora pauciflora Dalzell (1850) 136. - Type: Anonymous s.n. (K).

Canscora lawii Wight (1850) 1327. - Type: Ritchie 466 (lecto K, fide Klackenberg (1990); iso E), Belgium, Nord de Dharwur.

Canscora tenella Wight (1850) 1327. - Type: Wight s.n. (lecto K, fide Klackenberg (1990)), Malabar. 
Canscora divaricata Miq. ex C.B. Clarke (1885) 103. - Type: Hohenacker 670 (lecto TUB, designated here; iso E, G, K, MEL, S, U), India orientalis, prope Putore.

Canscora lancifolia Miq. ex C.B. Clarke (1885) 103. - Type: Hohenacker 305 (lecto TUB, designated here; iso BM, E, G, HBG, K, L, S, U), India orientalis, prope Mangalore.

Canscora kirkii N.E. Br. (1903) 558. - Type: Kirk s.n. (holo K), Zambia, Island at Victoria Falls, 1860.

Canscora khandalensis Santapau (1949) 485. - Type: Santapau 5015 (holo BLAT n.v.), Khandala, Bombay, 2 Oct. 1944.

Canscora diffusa (Vahl) R.Br. ex Roem. \& Schult. var. tetraptera Naik \& Pokle (1985) 673. - Type: Pokle 319 (n.v.), Aurangabad, Nagapur.

Canscora rubiflora X.X. Chen (1986) 177. - Type: Ban 26597 (holo GXMI n.v.), Guangxi, Tianlin, Xian, Anding Xiang, 17 Feb. 1959.

Plants 6-48 cm tall; stems basally and apically branched, with minute ridges up to 0.3 $\mathrm{mm}$ wide. Cauline leaves petiolate or rarely sessile, petiole up to $15 \mathrm{~mm}$ long, lamina with three main veins, elliptic, lanceolate to rarely ovate, 12-39 by 7-27 $\mathrm{mm}$, base attenuate to wedge-shaped, apex acute; lower cauline leaves deciduous. Inflorescences axillary, 3-40-flowered cymes; bracts linear or lanceolate to broadly ovate, 1-4 by 0.1-1.2 mm; pedicels $1.5-15 \mathrm{~mm}$ long. Calyx $3.8-10$ by $1-2.3 \mathrm{~mm}$, persistent, without calyx wings; tube $2.9-6.5 \mathrm{~mm}$ long; lobes triangular, acute, $1-2.1$ by $0.5-1.7 \mathrm{~mm}$ long. Corolla tube 4-7 mm long, pink, two lobes slightly larger than others, (broadly) elliptic, $1.4-5.6$ by $0.8-3 \mathrm{~mm}$, other two lobes elliptic $1.3-3.5$ by $0.7-1.8 \mathrm{~mm}$. Size of upper and lower stamens \pm equal. Filaments $0.4-1.4 \mathrm{~mm}$ long, not broadened, anthers $0.3-1.1$ by $0.3-0.4 \mathrm{~mm}$. Ovary $2.8-6.5$ by $0.5-2.8 \mathrm{~mm}$; style $0.6-4.5 \mathrm{~mm}$ long; stigmatic lobes $0.3-0.7$ by $0.2-0.5 \mathrm{~mm}$. Capsule $3-7$ by $1-3 \mathrm{~mm}$. Seeds irregular in shape, cubical to rectangular and with shallowly sunken sides, $0.25-0.33$ by $0.17-0.28$ $\mathrm{mm}$; outer testa reticulate, with shallow cells; testa cells irregularly polygonal, oriented in \pm linear rows; anticlinal walls straight.

Distribution - Tropical Africa, Asia and Australia.

Ecology - Moist places of rocky river banks, grasslands, mixed deciduous forests, roadsides; sandy, clayish soil. Sea level to $2000 \mathrm{~m}$ altitude. Flowering: throughout the year.

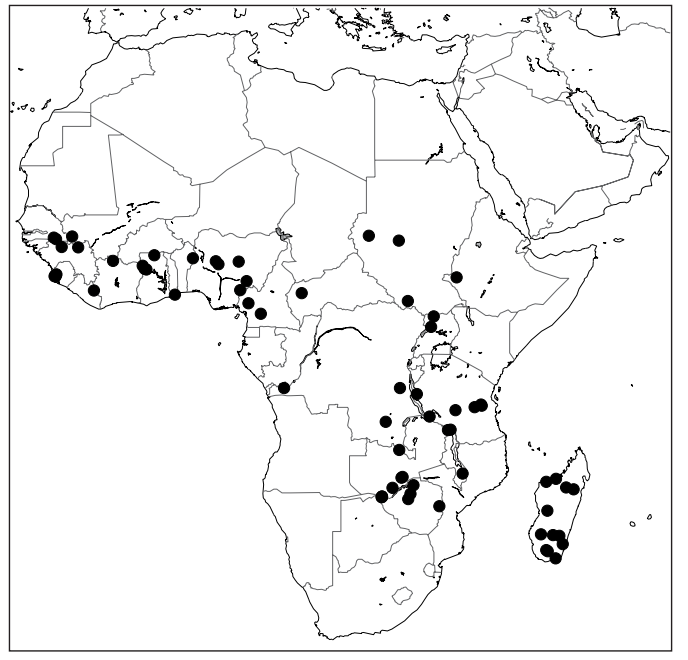

Map 5. Distribution of Canscora diffusa (Vahl) R.Br. ex Roem. \& Schult. in Africa. 


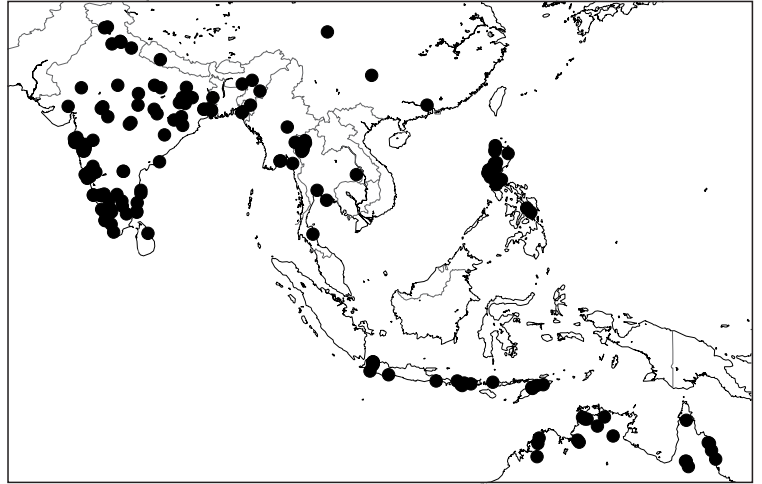

Map 6. Distribution of Canscora diffusa (Vahl) R.Br. ex Roem. \& Schult. in Asia and Australia.

Chromosome numbers $-2 \mathrm{n}=36,38$ (Christopher, 1976; Mallikarjuna et al., 1989).

Note - This species shows the widest distribution within the Canscorinae, ranging from tropical areas in Africa to Asia and Australia. It is also morphologically the most variable taxon of the subtribe. Several species (C. decurrens, $C$. divaricata, $C$. lancifolia and $C$. pauciflora) were described from India and are characterized by linear bracts and few-flowered inflorescences. Such individuals are found throughout the entire range of the species, sometimes even within collections with individuals with lanceolate to ovate leaves and many-flowered inflorescences. Consequently, these species are regarded as conspecific with $C$. diffusa. Canscora khandalensis from India was suggested to have larger flowers. This distinction, however, cannot be confirmed because the flower size range of $C$. khandalensis falls within that of $C$. diffusa. Canscora kirkii, an endemic from Southern Africa, was purported to differ from $C$. diffusa by having smaller flowers and many-flowered cymes. However, no clear boundary between these two taxa could be detected.

\subsection{Canscora heteroclita (L.) Gilg - Plate 3a, b; Map 7}

Canscora heteroclita (L.) Gilg (1895) 76. - Gentiana heteroclita L.(1767) 560 . - Exacum heteroclitum (L.) Willd. (1798) 639. - Type: Koenig s.n. (holo LINN, Photo no. 328.43).

Pladera sessiliflora Roxb. (1820) 417. - Canscora sessiliflora (Roxb.) Roem. \& Schult. (1827) 230. - Type: Roxburgh Ic. No. 1786 (K n.v.)

Plants $10-39 \mathrm{~cm}$ tall; stems basally and apically branched, with prominent wings up to $2.1 \mathrm{~mm}$ wide. Cauline leaves sessile, lamina with three main veins, ovate, $4-15$ by 3.4-11 mm, base truncate to shortly attenuate, apex acute. Inflorescences axillary, 1-6flowered cymes; bracts linear, slightly falcate, $1.5-4$ by $0.2-0.6 \mathrm{~mm}$. Flowers sessile. Calyx 8-9 by $1.5-1.6 \mathrm{~mm}$, persistent, without calyx wings; tube $6-7 \mathrm{~mm}$ long; lobes acute, 2 by $1 \mathrm{~mm}$. Corolla tube $6-9 \mathrm{~mm}$ long, rose, two lobes broadly elliptic, $3-5$ by $2.2-4 \mathrm{~mm}$, the other two lobes elliptic $1.2-2$ by $2.7-3 \mathrm{~mm}$. Filament of upper stamen 1.4-1.5 mm long, not broadened, anther $0.9-1.2$ by $0.4-0.5 \mathrm{~mm}$; filaments of lower stamens $1.1-1.9 \mathrm{~mm}$ long, anthers $1-1.4$ by $0.4-0.5 \mathrm{~mm}$. Ovary $4-4.3$ by $1-1.1 \mathrm{~mm}$; style up to $5.5 \mathrm{~mm}$ long; stigmatic lobes 0.8 by $0.7 \mathrm{~mm}$. Capsule 5.5 by $1.5 \mathrm{~mm}$. Seeds irregular in shape, cubical to rectangular and with shallowly sunken sides, c. 0.28 by 

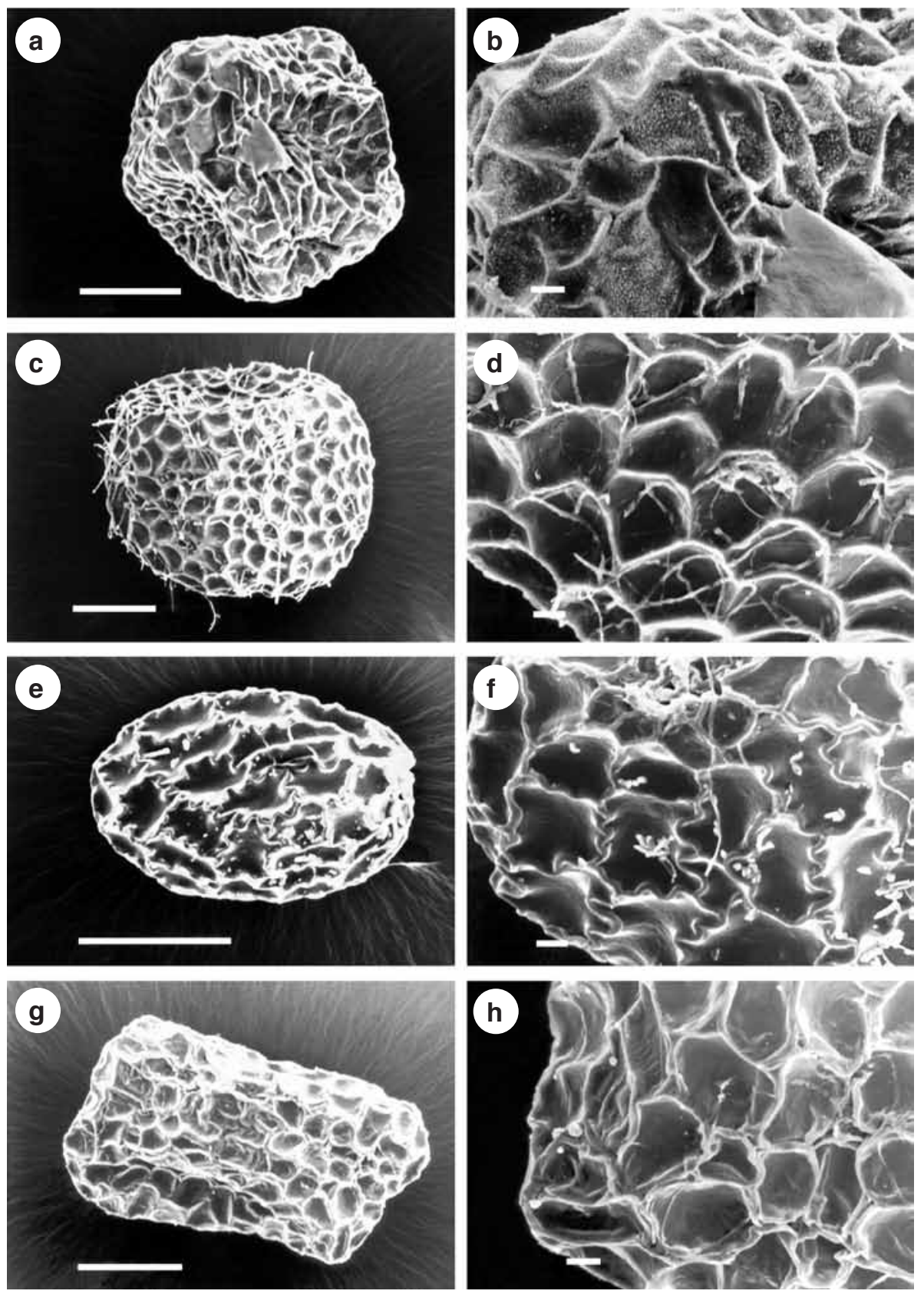

Plate 3. SEM photographs of seed coat structures. - a, b. Canscora heteroclita (L.) Gilg (Cramer 5129); c, d. C. schultesii Wall. ex Griseb. (Kurz 213); e, f. Hoppea dichotoma Willd. (Wood RHT 30759); g, h. H.fastigiata (Griseb.) C.B. Clarke (Jayasuriya 2092). - Scale bars: a, c, e, g = $100 \mu \mathrm{m}$; $\mathrm{b}, \mathrm{d}, \mathrm{f}, \mathrm{h}=10 \mu \mathrm{m}$. 


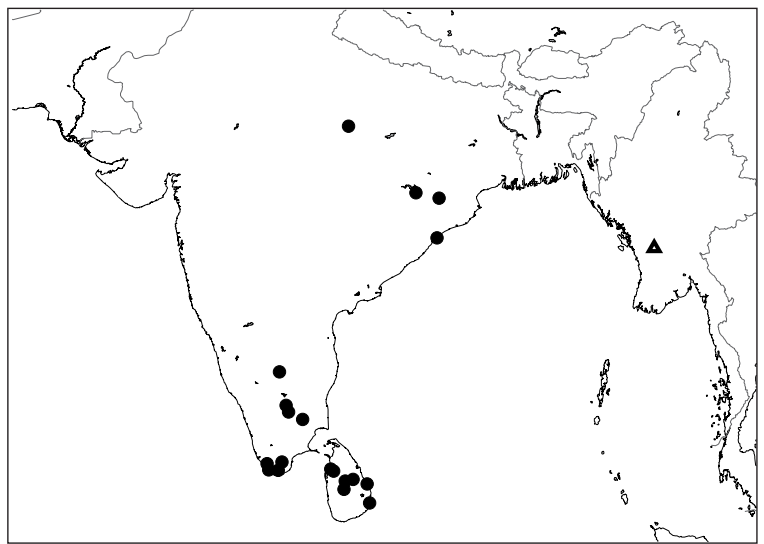

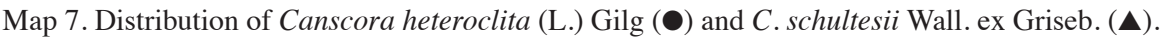

$0.26 \mathrm{~mm}$; outer testa reticulate, with shallow cells; testa cells irregularly polygonal, oriented irregularly; anticlinal walls straight.

Distribution - India and Sri Lanka.

Ecology - Moist places of open waste- and grassland, along ponds and streams, roadsides; sandy, heavy, rocky ground. Sea level to $700 \mathrm{~m}$ altitude. Flowering: throughout the year.

Chromosome number $-2 \mathrm{n}=38$ (Mallikarjuna et al., 1989).

\subsection{Canscora macrocalyx Miq. - Fig. 1; Plate 1e, f; Map 8}

Canscora macrocalyx Miq. (1856) 558. - Type: Horsfield s.n. (holo U), Java, Soerakarta.

Plants 8-39 cm tall; stems sparsely apically and partly basally branched, with prominent wings up to $1.5 \mathrm{~mm}$ wide. Cauline leaves sessile, lamina with three main veins, lanceolate, 5-18 by 3-9 $\mathrm{mm}$, base shortly attenuate, apex acute. Inflorescences axillary 1-5-flowered cymes; bracts lanceolate, 5-9 by 2-4.5 mm; pedicels 3-13 $\mathrm{mm}$ long. Calyx tube $10-13$ by $3-9 \mathrm{~mm}$ (incl. wings), persistent, with 4 prominent calyx

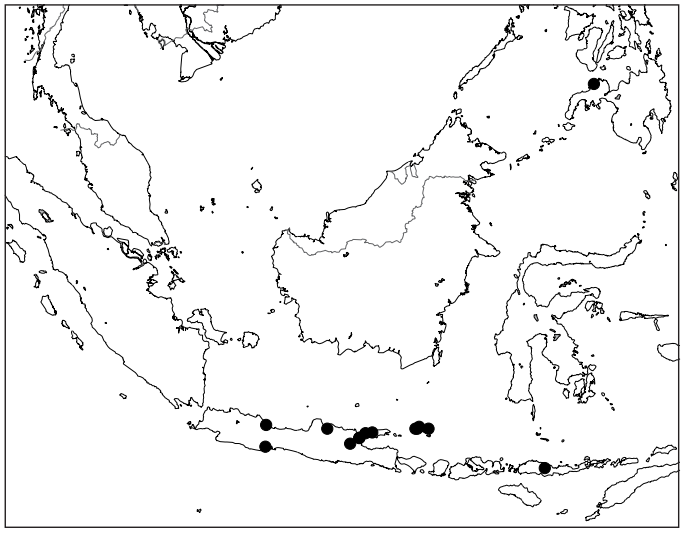

Map 8. Distribution of Canscora macrocalyx Miq. 
wings $1-3.5 \mathrm{~mm}$ wide; tube $7.5-11 \mathrm{~mm}$ long; lobes blunt with a point, 1 by $0.5 \mathrm{~mm}$. Corolla tube $9-11 \mathrm{~mm}$ long, white, two lobes broadly elliptic, $2-3$ by $3-4.5 \mathrm{~mm}$, the other two lobes elliptic 3 by $0.8-1.2 \mathrm{~mm}$. Filament of upper stamen $1.7-1.9 \mathrm{~mm}$ long, broadened below anther, anther 0.8 by $0.4 \mathrm{~mm}$; filaments of lower stamens $1.1-1.2$ $\mathrm{mm}$ long, anthers $0.8-0.9$ by $0.3-0.4 \mathrm{~mm}$. Ovary $6-7$ by $1.8-2.5 \mathrm{~mm}$; style up to 4.5 $\mathrm{mm}$ long; stigmatic lobes 0.8 by $0.3 \mathrm{~mm}$. Capsule $6-9$ by $2.2 \mathrm{~mm}$. Seeds irregular in shape, cubical to rectangular and with shallowly sunken sides, c. 0.35 by $0.26 \mathrm{~mm}$; outer testa reticulate, with shallow cells; testa cells irregularly polygonal, oriented irregularly; anticlinal walls straight.

Distribution - Java and Timor. One specimen was found on Mindanao (Philippines).

Ecology - Moist, open places in grasslands, fields; heavy soil. Sea level to $600 \mathrm{~m}$ altitude. Flowering: January to August.

\subsection{Canscora perfoliata Lam. - Plate 1a, b; Map 9}

Canscora perfoliata Lam. (1785) 601. - Type: Rheede tot Draakestein, Hortus indicus malabaricus 10: 103, f. 52 (1690).

Canscora ventricosa J.F. Gmel. (1791) 262, nom. superfl.

Canscora alata Wall. (1831) 4363, nom. nud.

Pladera alata Buch.-Ham. ex Wall. (1831) 4363B, nom. nud.

Canscora grandiflora Wight (1850) 1326. - Type: Jerdon s.n. (n.v.), Coorg and Western provinces of Mysore.

Canscora macrocalyx Miq. ex C.B. Clarke (1885) 104, nom. illeg., non Miq. (1855). - Type: Hohenacker 810 (lecto TUB, designated here; iso BM, E, G, K, S), India orientalis, Terra Canara et confin. In campis prope Mercara.

Plants $18-69 \mathrm{~cm}$ tall; stems basally and apically branched, with prominent wings up to $2.5 \mathrm{~mm}$ wide. Cauline leaves sessile, lamina with three main veins, elliptic to lanceolate, often falcate, $10-58$ by $5-39 \mathrm{~mm}$, base attenuate, apex acute. Inflorescences axillary, 1-3-flowered cymes; bracts orbicular, perfoliate, apex blunt or acute, $5-14$ by $5-15$ $\mathrm{mm}$; pedicels $1-10 \mathrm{~mm}$ long. Calyx $13-15$ by $7-8 \mathrm{~mm}$, persistent, with prominent four or eight wings $2-3.5 \mathrm{~mm}$ wide; tube $10-12 \mathrm{~mm}$ long; lobes anisomorphic, acute, two larger lobes 3 by $1 \mathrm{~mm}$ long, two shorter lobes 2 by $1 \mathrm{~mm}$. Corolla tube $13-14 \mathrm{~mm}$ long, white or cream, two lobes broadly elliptic, $8-9$ by $6-8 \mathrm{~mm}$, the other two lobes

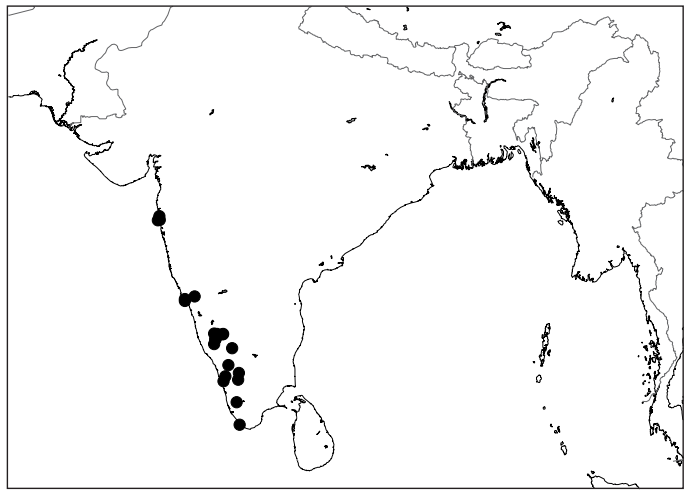

Map 9. Distribution of Canscora perfoliata Lam. 
elleptic, $5-6$ by $1.8-3 \mathrm{~mm}$. Filament of upper stamen $2.5-2.8 \mathrm{~mm}$ long, broadened below the anther, anther $1-1.8$ by $0.7-1 \mathrm{~mm}$; filaments of lower stamens $0.6-1.5 \mathrm{~mm}$ long, anthers $1.6-2.1$ by $0.5-0.7 \mathrm{~mm}$. Ovary $6-8$ by $1.8-2.5 \mathrm{~mm}$; style up to $9 \mathrm{~mm}$ long; stigmatic lobes 0.9 by $0.8 \mathrm{~mm}$. Capsule 7 by $2 \mathrm{~mm}$. Seeds irregular in shape, cubical to rectangular and with shallowly sunken sides, c. 0.40 by $0.36 \mathrm{~mm}$; outer testa reticulate, with shallow cells; testa cells irregularly polygonal, oriented irregularly; anticlinal walls straight.

Distribution - W India.

Ecology - Shady, wet places along roadsides and in deciduous forests. Altitude 400-1500 m. Flowering: October to March.

Chromosome number $-2 \mathrm{n}=38$ (Mallikarjuna et al., 1989).

\subsection{Canscora roxburghii Arn. ex Miq. - Fig. 2; Plate 1c, d; Map 3}

Canscora roxburghii Arn. ex Miq. (1852) 11. - Type: Roxburgh Ic. No. 1227 (K).

Canscora wallichii C.B. Clarke (1885) 105. - Type: Wallich 4365 (holo K-WALL, photo), Travancore.

Plants 6-54 cm tall; stems basally and apically branched, with prominent wings up to $1.4 \mathrm{~mm}$ wide. Cauline leaves sessile, lamina with three main veins, elliptic to lanceolate, slightly falcate, $10-38$ by $4-16 \mathrm{~mm}$, base attenuate, apex acute. Inflorescences axillary, 1-8-flowered cymes; bracts orbicular, perfoliate, $4-13$ by $5-13 \mathrm{~mm}$; pedicels $1-17 \mathrm{~mm}$ long. Calyx $8-9$ by 1-2 mm, persistent, without wings; tube $7 \mathrm{~mm}$ long; lobes acute, 2 by $1 \mathrm{~mm}$. Corolla tube $8-10 \mathrm{~mm}$ long, pale yellowish; two lobes broadly elliptic, $4.5-5$ by $2.7-3.7 \mathrm{~mm}$, the other two lobes elliptic $2.5-3$ by $1.2-1.5 \mathrm{~mm}$. Filament of upper stamen $1.7-1.9 \mathrm{~mm}$ long, broadened below anther, anther $0.7-0.9$ by $0.4-0.5$ $\mathrm{mm}$; filaments of lower stamens $0.9-1.5 \mathrm{~mm}$ long, anthers $0.9-1.2$ by $0.4-0.5 \mathrm{~mm}$. Ovary $4-5$ by $1-1.6 \mathrm{~mm}$; style up to $3.5 \mathrm{~mm}$ long; stigmatic lobes 0.5 by $0.3 \mathrm{~mm}$. Capsule 7 by $2.2 \mathrm{~mm}$. Seeds irregular in shape, cubical to rectangular and with shallowly sunken sides, c. 0.32 by $0.27 \mathrm{~mm}$; outer testa reticulate, with shallow cells; testa cells irregularly polygonal, oriented irregularly; anticlinal walls straight.

Distribution - S India and Sri Lanka.

Ecology - Damp roadside embankments among tall grass; gravelly loamy soil. Sea level to $2000 \mathrm{~m}$ altitude. Flowering: November to March.

Chromosome number $-2 \mathrm{n}=38$ (Mallikarjuna et al., 1989).

\subsection{Canscora schultesii Wall. ex Griseb. - Plate 3c, d; Map 7}

Canscora schultesii Wall. ex Griseb. (1839) 155. - Heterocanscora schultesii (Wall. ex Griseb.) C.B. Clarke (1875) 431. - Type: Wallich 4368 (lecto K, designated here; iso BM, E, G), Prome Hills, 1832.

Pladera pulchella Wall. (1831) 4368, nom. nud.

Plants 7-22 cm tall; stems basally branched, with ridges up to $0.4 \mathrm{~mm}$ wide. Rosette leaves sessile, lamina with three main veins, elliptic, $10-26$ by $5-14 \mathrm{~mm}$, base wedgeshaped to attenuate, apex obtuse or acute; cauline leaves sessile, linear to long lanceolate, $2.5-6$ by $0.4-0.6 \mathrm{~mm}$, apex acuminate. Inflorescences $6-20$-flowered spikes; bracts lanceolate, $9-11$ by $1.4-2.5 \mathrm{~mm}$. Flowers sessile. Calyx $6-6.8$ by $0.8-1.2 \mathrm{~mm}$, persistent, without calyx wings; tube $4.3-5.3 \mathrm{~mm}$ long; lobes taper-pointed, $1.2-1.8$ by 0.5 


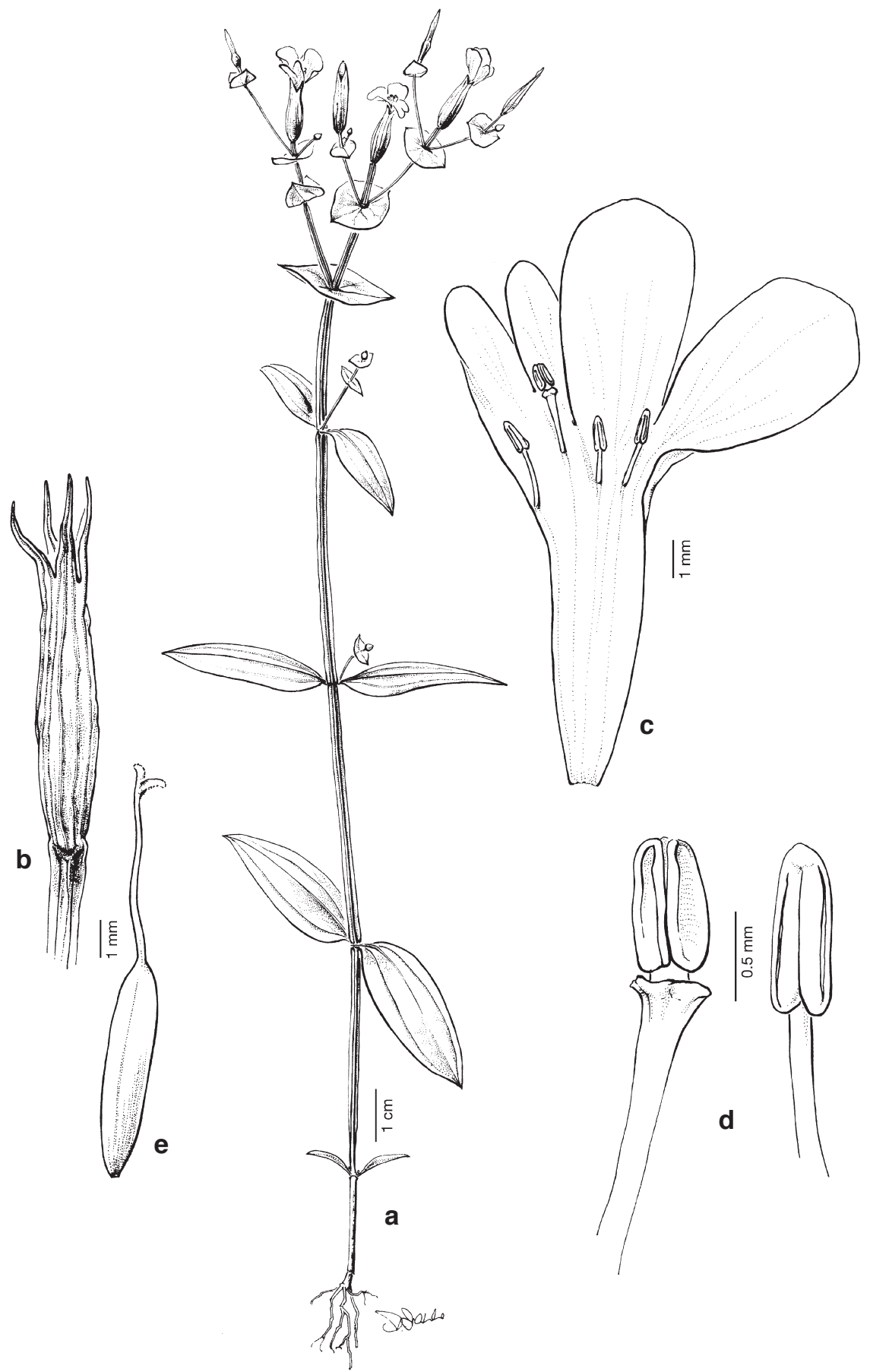

Fig. 2. Canscora roxburghii Arn. ex Miq. a. Habit; b. calyx; c. corolla with stamens; d. stamens of anisomorphic androecium; e. gynoecium (all: Barnes 1835). 
$\mathrm{mm}$. Corolla tube $8-9 \mathrm{~mm}$ long, colour unknown, two lobes broadly elliptic, 2.3-2.4 by $1.4-1.8 \mathrm{~mm}$, the other two lobes elliptic, $3.8-4$ by $1.5-1.8 \mathrm{~mm}$. Filament of upper stamen $1.4-1.7 \mathrm{~mm}$ long, not broadened, anther $1-1.1$ by $0.5-0.7 \mathrm{~mm}$; filaments of lower stamens $0.8-1 \mathrm{~mm}$ long, anthers $0.6-0.7$ by $0.4-0.5 \mathrm{~mm}$. Ovary $3.5-5$ by $1.2-1.3 \mathrm{~mm}$; style up to $3.5 \mathrm{~mm}$ long; stigmatic lobes $2.7-2.8$ by $0.6-0.8 \mathrm{~mm}$. Capsule 4.5 by $1.5 \mathrm{~mm}$. Seeds subglobose, c. 0.32 by $0.28 \mathrm{~mm}$; outer testa reticulate, testa cells polygonal; anticlinal walls curved.

Distribution - Burma.

Ecology - Unknown. Flowering: August.

Note - A rarely collected plant probably endemic to Burma.

\section{DOUBTFUL OR EXCLUDED SPECIES}

Canscora stricta Sedgw. (1921) 126. - Type: Sedgwick \& Bell 3393 (BLAT n.v.), Prov. Bombaiensis, North Canara, Castle Rock, 1600 m, Dec. 1917.

Plants up to $15 \mathrm{~cm}$ tall; stems sparsely branched, quadrangular. Cauline leaves sessile, lamina narrow lanceolate, 15 by $3 \mathrm{~mm}$, apex acute; leaves deciduous. Inflorescences axillary, 3-40-flowered cymes; bracts linear or subulate. Calyx 7 by $1.5 \mathrm{~mm}$ without calyx wings; tube $5 \mathrm{~mm}$ long; lobes linear $2 \mathrm{~mm}$ long. Corolla $10 \mathrm{~mm}$ long, pink; two lobes larger than others, orbicular, other two lobes oblong. Filament of upper stamen decurved apically, anthers $2 \mathrm{~mm}$ long. Ovary oblong. Capsule cylindric 3 by $1.5 \mathrm{~mm}$.

Distribution - NW India.

Ecology - On sheet rocks in forest clearings. Flowering: December.

Note - Unfortunately no specimens could be studied. The description of an anisomorphic androecium and a zygomorphic corolla indicates that $C$. stricta belongs to Canscora. Sedgwick's (1921) remark that also broadly ovate leaves occur, however, questions its taxonomic rank. Whether this taxon represents a good species or perhaps an atypic form of the morphologically variable $C$. diffusa can only be decided when sufficient material becomes available.

Canscora justicioides Griff. ex Voigt (1845) 520

Hitherto, no hints of the type collection could be found. Therefore, Griffith's collection 5807/1 (Z) representing Exacum tetragonum Roxb. has been chosen as neotype for the incomprehensively, but validly described Canscora justicioides Griff. ex Voigt.

\section{CRACOSNA Gagnep.}

Cracosna Gagnep. (1929) 776. - Type: Cracosna xyridiformis Gagnep. Canscora Lam. (1785) 601 p.p.

Annual, erect, glabrous herbs; stems apically sparsely branched, quadrangular, winged or with ridges. Rosette leaves present or absent; cauline leaves free, sessile, lamina with one main vein. Inflorescences axillary, few or many-flowered cymes; bracts free. Flowers tetramerous (except calyx in C.xyridiformis); sessile or pedicellate; with or without bracteoles. Calyx tetramerous, di- or trimerous in C.xyridiformis, tubular, winged or not, 

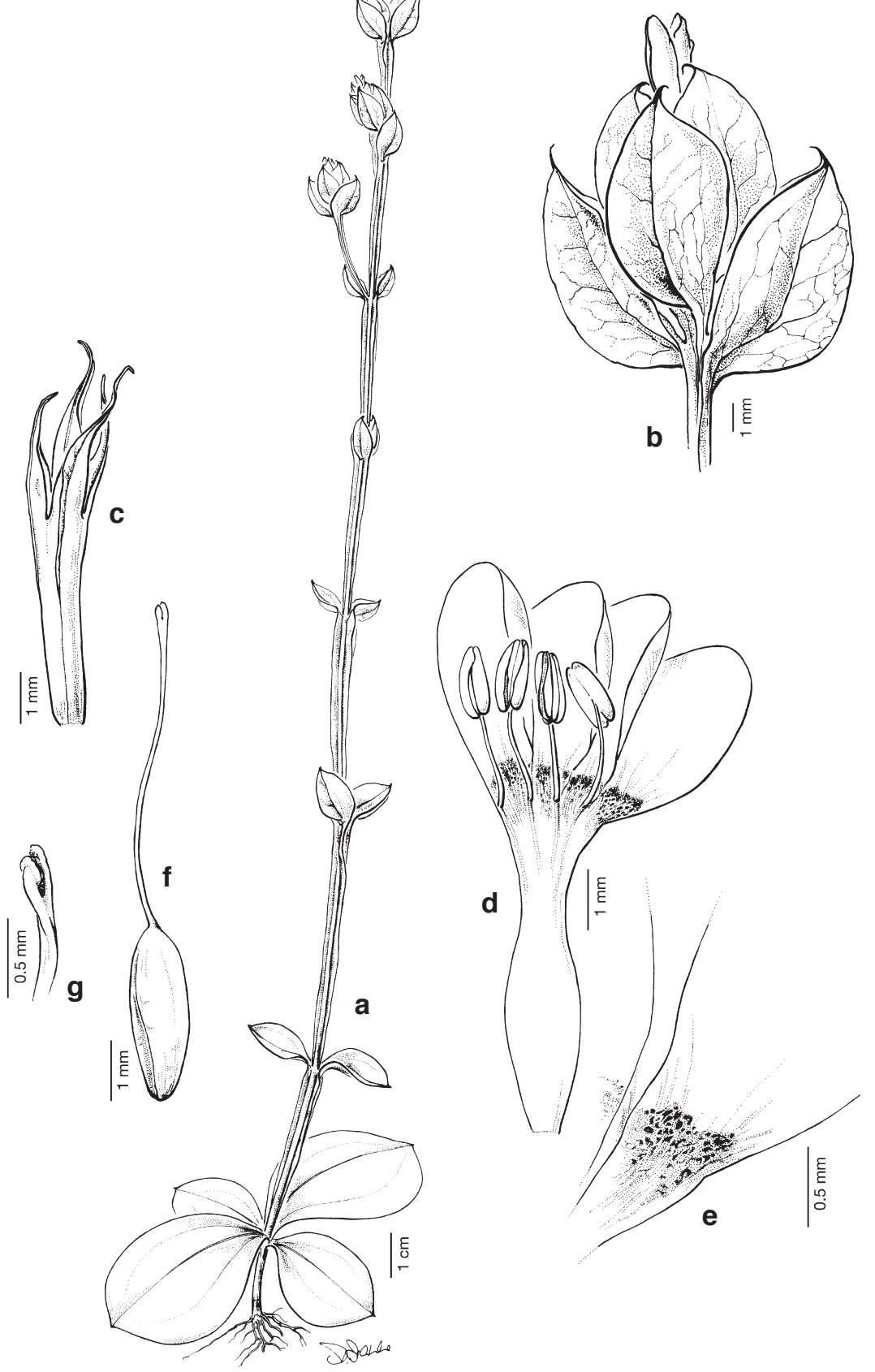

Fig. 3. Cracosna carinata (Dop) Thiv. a. Habit; b. flower; c. calyx; d. corolla with stamens; e. petal nectary; f. gynoecium; g. stigma (all: Chermsirivathana 1569). 
persistent. Corolla actinomorphic; salvershaped to tubular, mostly white or yellow, with nectaries between filaments; tube longer than lobes. Androecium isomorphic; inserted equally in upper part of corolla tube; anthers non-sagittate, persistent. Ovary oblong, ovoid or elliptic; stigmatic lobes oblong. Fruit an oblong capsule. Seeds irregular in shape, angular, often cubical to rectangular and with deeply sunken sides; outer testa reticulate, with shallow cells; testa cells polygonal, oriented irregularly; anticlinal walls prominent, mostly curved to straight; cuticle smooth.

Distribution - Three species in SE Asia.

\section{KEY TO THE SPECIES OF CRACOSNA}

1a. Flowers in lax panicles. Calyx lobes 4

2.2. C. gracilis

b. Flowers in dense heads. Calyx lobes 2,3 or $4 \ldots \ldots \ldots \ldots \ldots \ldots \ldots \ldots \ldots \ldots$

2a. Calyx lobes 2 or 3 . Bracts broadly rhomboid, not carinate. Cauline leaves $<4 \mathrm{~mm}$

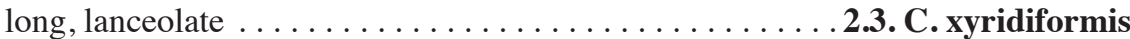

b. Calyx lobes 4 . Bracts falcate, carinate. Cauline leaves $>4 \mathrm{~mm}$ long, broadly lanceolate

2.1. C. carinata

2.1. Cracosna carinata (Dop) Thiv, comb. nov. - Fig. 3; Plate 4c, d; Map 10

Canscora carinata Dop (1912) 145. - Type: Massie s.n. (holo P-photo).

Plants 10-43 cm tall; stems with ridges up to $3.5 \mathrm{~mm}$ wide. Rosette leaves sessile, lamina ovoid to elliptic to obovoid, $4-38$ by $3-27 \mathrm{~mm}$, base wedge-shaped to attenuate, apex obtuse; cauline leaves broadly lanceolate to triangular, $4.8-16$ by $0.6-10$ $\mathrm{mm}$, apex acute to cuspidate. Inflorescences axillary, 1-7-flowered head-like cymes; bracts carinate, $5-10$ by $2.1-6.2 \mathrm{~mm}$, with wings up to $1.5 \mathrm{~mm}$ wide. Flowers sessile, without bracteoles. Calyx 5-7 by $1.2-1.5 \mathrm{~mm}$, with wings up to $0.3 \mathrm{~mm}$ wide; tube

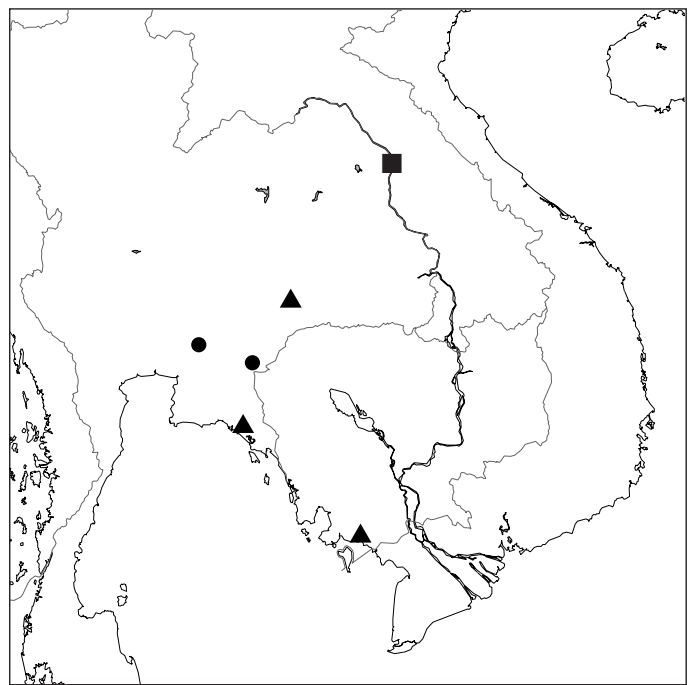

Map 10. Distribution of Cracosna carinata (Dop) Thiv $(\bullet)$, C. gracilis (Dop) Thiv (ם) and C.xyridiformis Gagnep. (山). 

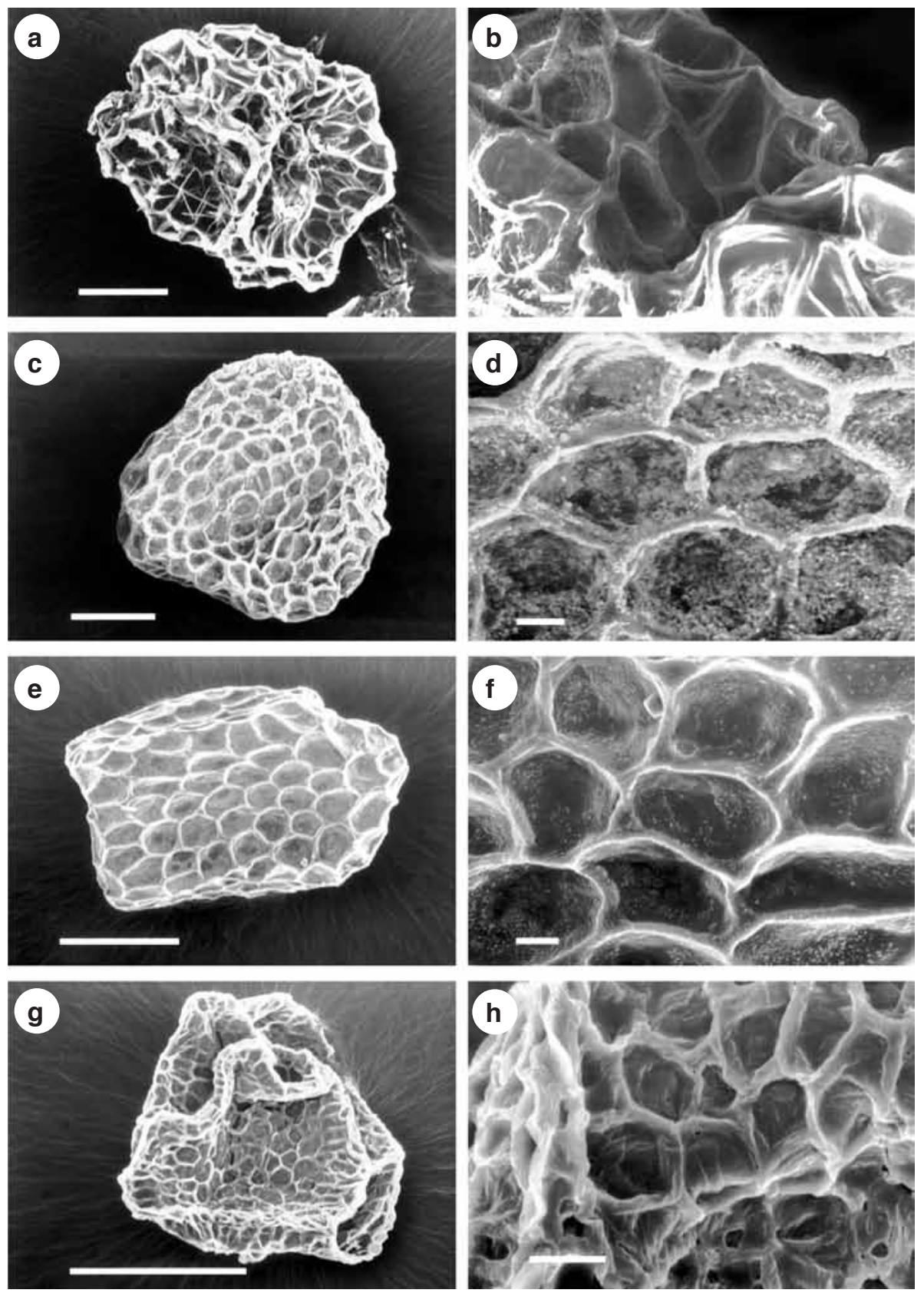

Plate 4. SEM photographs of seed coat structures. - a, b. Schinziella tetragona (Schinz) Gilg (Champluvier 5136); c, d. Cracosna carinata (Dop) Thiv (Chermsirivathana 1569); e, f. C. gracilis (Dop) Thiv (Smitinand 8438); g, h. C.xyridiformis Gagnep. (Kerr 8226). - Scale bars: a, c, e, g = $100 \mu \mathrm{m}$; $\mathrm{b}, \mathrm{d}, \mathrm{f}, \mathrm{h}=10 \mu \mathrm{m}$. 
2.8-3.8 mm long; lobes pungent, $2.2-3.3$ by $0.6-0.8 \mathrm{~mm}$. Corolla $8-9.5 \mathrm{~mm}$ long, white; lobes broadly lanceolate to oblong, $3.1-3.8$ by $1-1.8 \mathrm{~mm}$. Filaments $1.1-1.4$ mm long; anthers $0.9-1.5$ by $0.4-0.6 \mathrm{~mm}$. Ovary oblong to long obovoid, $3.2-3.3$ by $0.8-1 \mathrm{~mm}$; style up to $4 \mathrm{~mm}$ long; stigmatic lobes oblong 0.4 by $0.2 \mathrm{~mm}$. Capsule 3.5 by $1.7 \mathrm{~mm}$. Seeds irregular in shape, with deeply sunken sides, c. 0.34 by $0.30 \mathrm{~mm}$.

Distribution - Thailand and Laos (Dop, 1912).

Ecology - Dry soil. Flowering: October to November.

\subsection{Cracosna gracilis (Dop) Thiv, comb. nov. - Plate 4e, f; Map 10}

Canscora gracilis Dop (1912) 146. - Type: Counillon s.n. (holo P-photo).

Plants 10-25 cm tall; stems with ridges up to $0.6 \mathrm{~mm}$ wide. Rosette leaves sessile, lamina elliptic, $7-18$ by $4-12 \mathrm{~mm}$, base attenuate, apex obtuse to acute; cauline leaves lamina linear to long lanceolate, $2-4$ by $0.4-0.7 \mathrm{~mm}$, apex acute. Inflorescences $1-6$ flowered panicle-like inflorescences; bracts linear, $2-2.5$ by $0.2-0.3 \mathrm{~mm}$. Flowers pedicellate, pedicels 2-4 mm long, with two linear bracteoles up to $2 \mathrm{~mm}$ long. Calyx $3.8-4.5$ by $1-1.3 \mathrm{~mm}$ with four ridges; tube $2-2.4 \mathrm{~mm}$ long; lobes taper-pointed, $1.5-2$ by $0.3-0.5 \mathrm{~mm}$ long. Corolla $5.5-7 \mathrm{~mm}$ long, colour unknown; lobes long elliptic to lanceolate, $1.8-2.5$ by $4-5 \mathrm{~mm}$. Filaments $0.8-1.3 \mathrm{~mm}$ long; anthers 0.3 by $0.2 \mathrm{~mm}$. Ovary oblong, 3-4 by $0.7 \mathrm{~mm}$; style up to $2 \mathrm{~mm}$ long; stigmatic lobes small oblong 0.2 by $0.1 \mathrm{~mm}$. Capsule $3-4$ by $1 \mathrm{~mm}$. Seeds cubical to rectangular, c. 0.27 by $0.18 \mathrm{~mm}$.

Distribution - Thailand and Laos.

Ecology - Dry dipterocarp forests. Altitude 200 m. Flowering: December.

Note - Due to their isomorphic androecia, Canscora carinata and Canscora gracilis were transferred to Cracosna.

\subsection{Cracosna xyridiformis Gagnep. - Plate 4g, h; Map 10}

Cracosna xyridiformis Gagnep. (1929) 776. - Type: Geoffroy 461 (lecto P-photo, fide Hul (in press)), Cambodge, Kep, Province de Kampot, 28 Oct. 1904. Syntypes fide Hul (in press): Massie s.n. (P-photo), Laos; Bois 2174 (n.v.); Pierre s.n. (n.v.).

Herb $18-45 \mathrm{~cm}$ tall; stems with minute $0.1 \mathrm{~mm}$ wide ridges. Rosette leaves probably absent or early deciduous; cauline leaves minute, lanceolate, $2-3.5$ by $0.3-0.5 \mathrm{~mm}$, apex acute. Inflorescences axillary, 3-30-flowered head-like cymes; bracts broadly rhomboid, $4.7-6$ by $2.9-4.9 \mathrm{~mm}$, without wings. Flowers (except calyx) tetramerous, sessile, without bracteoles. Calyx di- or trimerous, 4 by $1.5 \mathrm{~mm}$, without calyx wings; tube 2-2.8 mm long; lobes obovoid, $1.2-2$ by $0.8-1.1 \mathrm{~mm}$, apex acute. Corolla $6-6.5$ $\mathrm{mm}$ long, yellow; lobes broadly lanceolate, $2.2-2.5$ by $1-2 \mathrm{~mm}$. Filaments up to $1 \mathrm{~mm}$ long; anthers 0.7 by $0.3 \mathrm{~mm}$. Ovary ovoid to elliptic, $3-3.2$ by $1.1 \mathrm{~mm}$; style bifid, up to $2.5 \mathrm{~mm}$ long; stigmatic lobes oblong 1.1 by $0.2 \mathrm{~mm}$. Capsule 3.5 by $1.2 \mathrm{~mm}$. Seeds irregular in shape, with deeply sunken sides, c. 0.16 by $0.14 \mathrm{~mm}$.

Distribution - Thailand, Cambodia, Laos (Gagnepain, 1929).

Ecology - Open grassland in deciduous forests. Sea level to $100 \mathrm{~m}$ altitude. Flowering: September to January. 

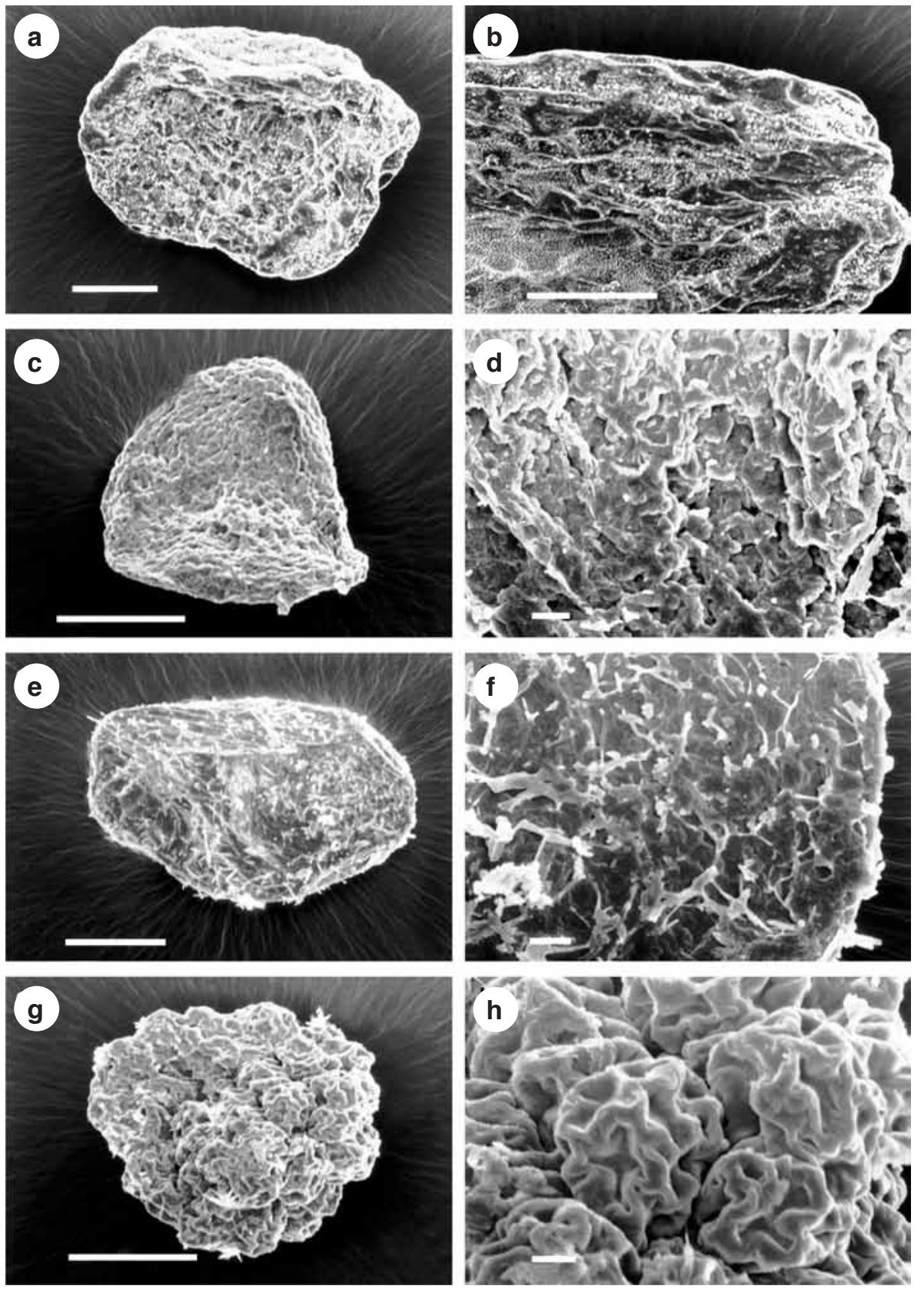

Plate 5. SEM photographs of seed coat structures. - a, b. Microrphium pubescens C.B. Clarke subsp. pubescens (Hansen 12355); c, d. Duplipetala pentanthera (C.B. Clarke) Thiv (Meyer 9642); e, f.D. hexagona (Kerr) Thiv (Van Beusekom 3814); g, h. Phyllocyclus helferianus Kurz (Niyomdham 436). - Scale bars: a, c, e, $\mathrm{g}=100 \mu \mathrm{m} ; \mathrm{b}, \mathrm{d}, \mathrm{f}, \mathrm{h}=10 \mu \mathrm{m}$. 


\section{DUPLIPETALA Thiv, gen. nov.}

Genus novus Phyllocyclus Kurz similis sed differt foliis caulinis libris, corolla hexamera et basi fili tenui. - Typus: Duplipetala pentanthera (C.B. Clarke) Thiv.

Canscora Lam. (1785) 601 p.p.

Perennial or annual, erect, glabrous herbs; stems apically and partly basally branched, terete sometimes with minute ridges. Rosette leaves absent; cauline leaves free, petiolate, lamina with one main vein, lanceolate to ovate, sometimes anisophyllous, base attenuate, apex acute. Inflorescences axillary, few-flowered, lax cymes; bracts perfoliate or free. Flowers hexa- or rarely pentamerous (except calyx); pedicellate; without bracteoles. Calyx tri- or hexamerous, inflated urceolate with reticulate venation, with unconnected wings, persistent; lobes triangular, apex acute to blunt with a point. Corolla actinomorphic, tubular to salvershaped, white-cream-coloured; tube longer than lobes; lobes broadly lanceolate, apex obtuse to cuspidate. Androecium isomorphic or anisomorphic with filaments of different length; inserted almost equally in central part of corolla tube; anthers sagittate, deciduous. Ovary oblong to obovoid, surface lacunose; stigmatic lobes rounded. Fruit an oblong capsule. Seeds irregular in shape, subglobose to angular with shallowly to deeply sunken sides; testa cells irregular in shape, oriented irregularly; cuticle with scurfy exudates.

Distribution - Two species in SE Asia.

Etymology - The name refers to the occurrence of six petals and three sepals in D. pentanthera.

Note - With its isomorphic or anisomorphic androecium with filaments of different length, Duplipetala is clearly distinct from Canscora s.str. It can be distinguished from Phyllocyclus by its free cauline leaves, mostly hexamerous corolla, a reticulate calyx venation and usually unbroadened filaments.

\section{KEY TO THE SPECIES OF DUPLIPETALA}

1a. Calyx lobes three. Plants perennial. Bracts mostly perfoliate 3.2. D. pentanthera

b. Calyx lobes six. Plants annual. Bracts mostly free ........ 3.1. D. hexagona

\subsection{Duplipetala hexagona (Kerr) Thiv, comb. nov. - Plate 5e, f; Map 11}

Canscora hexagona Kerr (1940) 181. - Type: Kerr 8059 (lecto BM, designated here; iso E, K, L), Chan Tuk, Korat, 300 m, 18 Dec. 1923.

Annual herbs $12-47 \mathrm{~cm}$ tall; stems with minute ridges up to $0.2 \mathrm{~mm}$ wide. Cauline leaves slightly petiolate; petiole up to $0.5 \mathrm{~mm}$ long; lamina $13-70$ by $5-31 \mathrm{~mm}$. Inflorescences axillary, 3-8-flowered cymes; bracts lanceolate to linear, free, 1.7-5 by $0.7-2.1 \mathrm{~mm}$. Flowers hexamerous; pedicels up to $3 \mathrm{~mm}$ long. Calyx urceolate $6.5-8$ by 5-7.8 $\mathrm{mm}$, with six wings up to $1 \mathrm{~mm}$ wide; tube 5.5-6.5 mm long; lobes $1.3-1.6$ by $1.1-2 \mathrm{~mm}$, with glands on its lower parts. Corolla tubular, 7-7.5 mm long; lobes $1.8-2$ by $0.9-1.2 \mathrm{~mm}$. Stamens mostly inserted at equal levels, filaments sometimes of different length, $2.5-3.8 \mathrm{~mm}$ long, anthers $0.7-0.9$ by $0.5-0.6 \mathrm{~mm}$. Ovary oblong to obovoid, surface constricted, $2.8-3.3$ by $1.7-2 \mathrm{~mm}$; style up to $4.3 \mathrm{~mm}$ long; stigmatic lobes 0.4 by $0.4 \mathrm{~mm}$. Capsule 4.5 by $2.5 \mathrm{~mm}$. Seeds c. 0.27 by $0.20 \mathrm{~mm}$. 


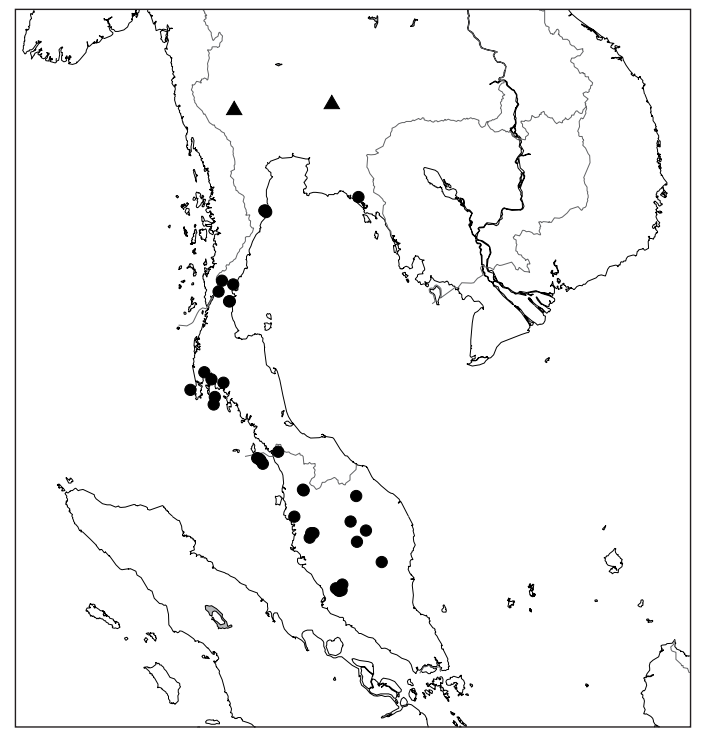

Map 11. Distribution of Duplipetala hexagona (Kerr) Thiv (A) and D. pentanthera (C.B. Clarke) Thiv $(\bullet)$.

Distribution - Thailand.

Ecology - Open, fire influenced, dry dipterocarp forests, savannahs; limestone rocks. Altitude 400-1300 m. Flowering: November to December.

Note - Based on its free cauline leaves and its flower morphology, Canscora hexagona was transferred to Duplipetala.

3.2. Duplipetala pentanthera (C.B. Clarke) Thiv, comb. nov. - Fig. 4; Plate 5c, d; Map 11

Canscora pentanthera C.B. Clarke (1906) 89. - Type: Ridley 8214 (holo K), Selangor, Gua Batu, limestone rocks, 1897.

Perennial, suffrutescent herbs $24-52 \mathrm{~cm}$ tall. Cauline leaves petiolate; petiole 9-44 mm long; lamina $38-95$ by $16-36 \mathrm{~mm}$. Inflorescences axillary, 2-20-flowered cymes; bracts broadly ovate, mostly perfoliate, $5-13$ by $5-12 \mathrm{~mm}$. Flowers (except calyx) hexa-, rarely pentamerous, pedicels up to $2 \mathrm{~mm}$ long. Calyx trimerous, $10-12$ by $4-8$ $\mathrm{mm}$, with three wings up to $1.5 \mathrm{~mm}$ wide; tube $7-9 \mathrm{~mm}$ long; lobes $2.1-2.2$ by $2.5-4$ $\mathrm{mm}$. Corolla salvershaped to tubular, $11.8-14 \mathrm{~mm}$ long; lobes $3-3.2$ by $1.7-1.8 \mathrm{~mm}$. Filaments sometimes of different length, $2-5 \mathrm{~mm}$ long; anthers $1.8-2$ by $0.8-1 \mathrm{~mm}$. Ovary oblong, surface constricted, $3.8-5$ by $1.1-1.6 \mathrm{~mm}$; style up to $7 \mathrm{~mm}$ long; stigmatic lobes 0.3 by $0.4 \mathrm{~mm}$. Capsule 6 by $3 \mathrm{~mm}$. Seeds c. 0.21 by $0.19 \mathrm{~mm}$.

Distribution - Thailand and W Malaysia.

Ecology - Tropical forests, limestone rocks. Sea level to $1300 \mathrm{~m}$ altitude. Flowering: throughout the year. 


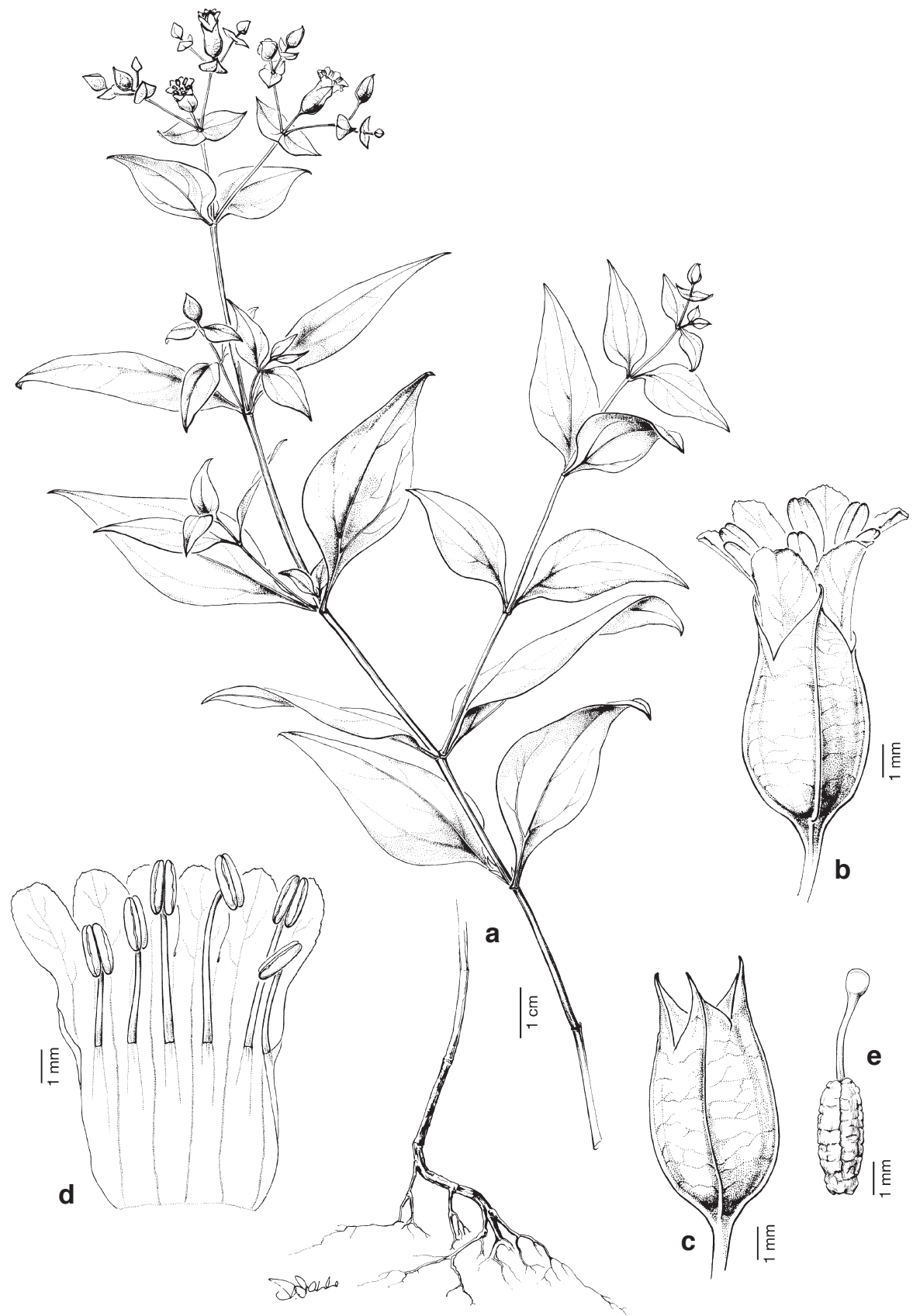

Fig. 4. Duplipetala pentanthera (C.B. Clarke) Thiv. a. Habit; b. flower; c. calyx; d. corolla with stamens; e. gynoecium (all: Meyer 9642). 


\section{HOPPEA Willd.}

Hoppea Willd. (1801) 434. - Type: Hoppea dichotoma Willd.

Pladera Sol. ex Roxb. (1820) 416 p.p.

Annual, erect, glabrous herbs; stems quadrangular, strongly winged. Rosette leaves absent; cauline leaves free, sessile; lamina with one main vein, base shortly attenuate to wedge-shaped, apex obtuse or cuspidate. Inflorescences axillary, many-flowered dense or lax cymes; bracts free. Flowers tetramerous, sessile or pedicellate, without bracteoles. Calyx funnelshaped to campanulate, with two ridges at the margin of each sepal, persistent; lobes triangular to blunt with a point. Corolla actinomorphic, tubular to funnelshaped, white; tube longer than lobes; lobes broadly elliptic. Androecium anisomorphic (Fig. 5c), stamens inserted in upper or lower part of corolla tube; one or rarely two upper stamens with longer filament and larger anther than the remaining lower stamens; anthers sagittate, persistent. Ovary obovoid to oblong elliptic; stigmatic lobes rounded to oblong. Fruit an obovoid to oblong elliptic capsule. Seeds elliptic to cubical to rectangular; outer testa reticulate, with shallow cells; testa cells irregularly polygonal, oriented irregularly; anticlinal walls prominent, mostly curved or undulating; cuticle smooth.

Distribution - Two species mainly on the Indian subcontinent.

\section{KEY TO THE SPECIES OF HOPPEA}

1a. Stamens inserted in the middle of the corolla tube. At least some flowers pedicellate. Stems basally and apically branched. Cauline leaves ovate . . . . . . . .

4.1. H. dichotoma

b. Stamens inserted in the upper part of the corolla tube close to the sinuses. Flowers sessile. Stems mostly only apically branched. Cauline leaves ovate to rhomboid .

4.2. H. fastigiata

\subsection{Hoppea dichotoma Willd. - Plate 3e, f; Map 12}

Hoppea dichotoma Willd. (1801) 434. - Type: Klein s.n. (holo B-W), India orientalis, 1800.

Pladera pusilla Roxb. (1814) 10. - Type: Wallich 4366 (holo K-WALL, photo).

Hoppea dichotoma Willd. forma pedicellata S.R. Paul (1977) 183. - Type: Paul 96213 A (n.v.),

Sadni, Netarhat, 28 Dec. 1972.

Plants $1.5-10 \mathrm{~cm}$ tall; stems basally and apically branched, with wings up to $0.5 \mathrm{~mm}$ wide. Cauline leaves ovate, $2.3-7$ by $1.2-4 \mathrm{~mm}$, base shortly attenuate, apex obtuse to cuspidate. Inflorescences axillary, 6-30-flowered lax dichasia; bracts linear to longlanceolate, $1.8-5$ by $0.3-1.6 \mathrm{~mm}$. Flowers pedicellate or sessile, pedicels up to $3(-7)$ $\mathrm{mm}$ long. Calyx funnelshaped to campanulate, $2.3-3.1$ by $1.3-2 \mathrm{~mm}$; tube $1.4-2 \mathrm{~mm}$ long; lobes $1.1-1.6$ by $0.6-1 \mathrm{~mm}$. Corolla tube $2.5-3 \mathrm{~mm}$ long; lobes $1-1.4$ by $0.5-0.7$ $\mathrm{mm}$. Stamens inserted in the middle part of the corolla tube; filament of upper stamen $0.4-0.5 \mathrm{~mm}$ long, anther $0.2-0.3$ by $0.2 \mathrm{~mm}$; filaments of the lower stamens $0.1-0.3$ $\mathrm{mm}$ long, anthers 0.1 by $0.1 \mathrm{~mm}$. Ovary $0.8-1.1$ by $0.5-0.9 \mathrm{~mm}$; style up to $0.2 \mathrm{~mm}$ long; stigmatic lobes oblong, c. 0.1 by $0.1 \mathrm{~mm}$. Capsule 1.5 by $1.3 \mathrm{~mm}$. Seeds elliptic, c. 0.21 by $0.14 \mathrm{~mm}$; anticlinal walls undulating. 


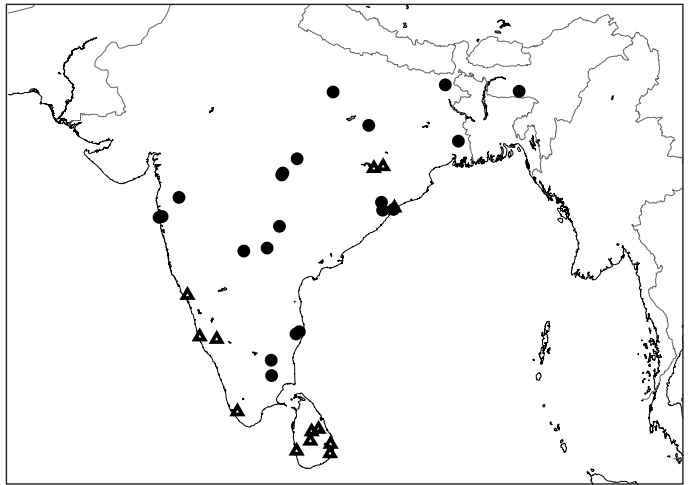

Map 12. Distribution of Hoppea dichotoma Willd. (•) and H. fastigiata (Griseb.) C.B. Clarke ( $\mathbf{\Delta})$. Occurrences of H. dichotoma in Africa and the Philippines are not shown.

Distribution - India. Probably introduced in the Philippines (Luzon), Senegal (Berhaut, 1975) and Ethiopia (Raynal, 1967).

Ecology - Open places of cultivated fields, roadsides and forests; $300-1800 \mathrm{~m}$ altitude. Flowering: October to March.

Notes - There are several arguments to regard the occurrence of H. dichotoma in Africa and the Philippines as not autochthonic: 1. Its sister species, H. fastigiata (Thiv \& Kadereit, 2002) is restricted to India and Sri Lanka; 2. outside of India there were only a few specimens collected; 3. Hoppea grows as a weed in India, indicative of their potential for introduction; 4. the populations outside of India are very isolated.

The structure of vascular bundles of this species was studied by Kshetrapal (1973).

Chromosome number $-2 \mathrm{n}=38$ (Mallikarjuna et al., 1989).

\subsection{Hoppea fastigiata (Griseb.) C.B. Clarke - Fig. 5; Plate 3g, h; Map 12}

Hoppea fastigiata (Griseb.) C.B. Clarke (1885) 100. - Cicendia fastigiata Griseb. (1839) 158. - Pladera fastigiata (Griseb.) C.B. Clarke (1875) 429. - Type: Macrae s.n. (lecto K, designated here), Ceylon, Colombo, in fields.

Plants 1-12 cm tall; stems mostly apically branched, with wings up to $1 \mathrm{~mm}$ wide. Cauline leaves ovate to rhomboid, $3.2-11$ by $1.8-7 \mathrm{~mm}$, base wedge-shaped to attenuate, apex obtuse to cuspidate. Inflorescences axillary, 3-20-flowered dense cymes; bracts linear to long lanceolate, $2.5-4.5$ by $0.7-1.8 \mathrm{~mm}$. Flowers sessile. Calyx long funnelshaped, $2.5-3.8$ by $1.4-1.9 \mathrm{~mm}$; tube $1.5-2 \mathrm{~mm}$ long; lobes $1.3-1.6$ by 0.8 $\mathrm{mm}$. Corolla tube 2-2.3 mm long; lobes $1-1.4$ by $0.5-0.7 \mathrm{~mm}$. Stamens inserted in the upper part of the corolla tube; filament of upper stamen $0.3-0.4 \mathrm{~mm}$ long, anther $0.3-0.4$ by $0.3-0.5 \mathrm{~mm}$; filaments of lower stamens $0.1-0.2 \mathrm{~mm}$ long, anthers $0.2-0.3$ by $0.1-0.2 \mathrm{~mm}$. Ovary $1.2-2.2$ by $0.7-1.3 \mathrm{~mm}$; style up to $0.8 \mathrm{~mm}$ long; stigmatic lobes oblong, 0.3 by $0.2 \mathrm{~mm}$. Capsule 2.5 by $1.3 \mathrm{~mm}$. Seeds cubical to rectangular, c. 0.35 by $0.19 \mathrm{~mm}$; anticlinal walls curved.

Distribution - India and Sri Lanka.

Ecology - Moist patches of grasslands and secondary forests. Sea level to $1700 \mathrm{~m}$ altitude. Flowering: throughout the year.

Chromosome number $-2 \mathrm{n}=38$ (Mallikarjuna et al., 1989). 

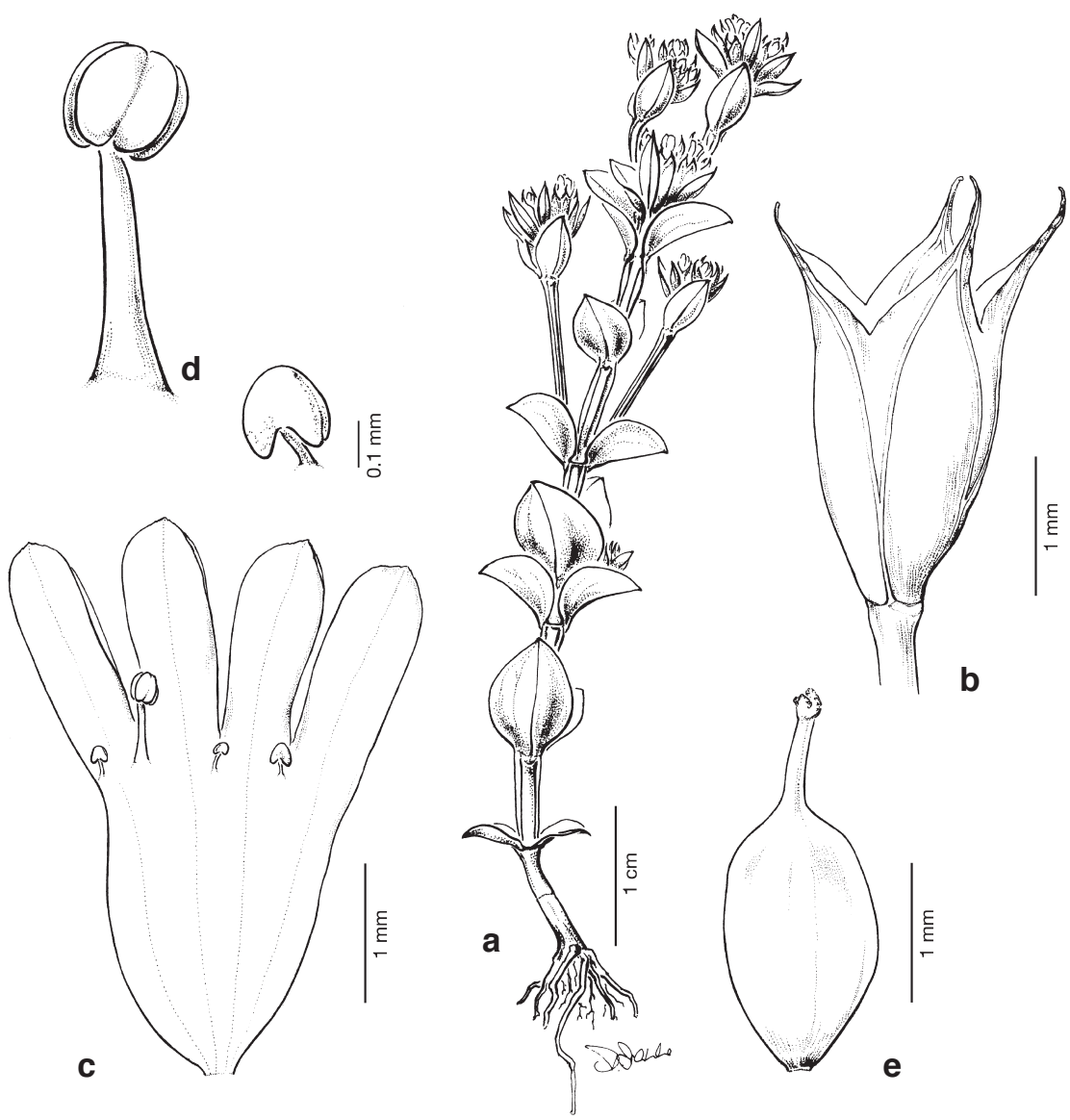

Fig. 5. Hoppea fastigiata (Griseb.) C.B. Clarke. a. Habit; b. calyx; c. corolla with stamens; d. stamens of anisomorphic androecium; e. gynoecium (all: Cramer 5143).

\section{MICRORPHIUM C.B. Clarke}

Microrphium C.B. Clarke (1906) 88. - Type: Microrphium pubescens C.B. Clarke.

Perennial, erect, suffrutescent herbs with bristles on all green parts; stems mostly apically branched, terete without ridges. Rosette leaves absent; cauline leaves free, petiolate; lamina with one main vein, lanceolate to ovate, base attenuate to wedge-shaped, apex acute. Inflorescences axillary, many-flowered, lax monochasia; bracts free. Flowers pentamerous (except calyx), pedicellate, without bracteoles. Calyx dimerous, tubular, without wings, persistent; two triangular lobes, apex acute to blunt with a point. Corolla actinomorphic, salvershaped, white-cream-coloured; tube longer than lobes. Androecium isomorphic; stamens inserted equally in upper part of corolla tube; anthers sagittate, deciduous. Ovary obovoid; stigmatic lobes minute. Fruit an obovoid to oblong capsule. Seeds irregular in shape, subglobose to angular with shallowly sunken sides; 
outer testa irregular in shape, with shallow cells; testa cells irregular in shape, oriented irregularly; anticlinal walls straight; cuticle with scaly exudates.

Distribution - One species in SE Asia.

\subsection{Microrphium pubescens C.B. Clarke}

Microrphium pubescens C.B. Clarke (1906) 88. - Type: Curtis 2528 (K n.v.), Kedah.

Microrphium elmerianum Regalado \& Soejarto (1997) 77. - Type: Soejarto et al. 6499 (holo F; iso A n.v., PHN n.v.), Philippines, Palawan Island, Puerto Princesa, Bacungan hills, slopes above Nagtabon beach, 9 51' N, 118 37' E, 50-100 m, 9 April 1989.

Plants $35-150 \mathrm{~cm}$ tall. Cauline leaves petiolate; petiole 2-48 $\mathrm{mm}$ long; lamina $30-140$ by $14-46 \mathrm{~mm}$. Inflorescences axillary, 12-30-flowered, \pm erect or pendulous lax monochasia; bracts broadly obovoid to elliptic, $3-9.7$ by $1.7-4 \mathrm{~mm}$; pedicels $2-12 \mathrm{~mm}$ long. Calyx $4-4.5$ by $1.9-2.1 \mathrm{~mm}$; tube $3-3.2 \mathrm{~mm}$ long; lobes $1-1.3$ by $2 \mathrm{~mm}$. Corolla $4.8-5.2 \mathrm{~mm}$ long; lobes elliptic, $2.2-3.2$ by $0.9-1 \mathrm{~mm}$, apex obtuse to cuspidate. Filaments $0.2-1.8 \mathrm{~mm}$ long; anthers $1.9-2.2$ by $0.5-0.6 \mathrm{~mm}$. Ovary $1.9-2.2$ by $1.1-1.3 \mathrm{~mm}$; style up to $3.3 \mathrm{~mm}$ long; stigmatic lobes 0.2 by $0.1 \mathrm{~mm}$. Capsule 4 by $2.5 \mathrm{~mm}$. Seeds $0.35-0.4$ by $0.27 \mathrm{~mm}$.

Ecology - Tropical forests; limestone rocks. Sea level to $260 \mathrm{~m}$ altitude.

\section{KEY TO THE SUBSPECIES OF MICRORPHIUM PUBESCENS}

1a. Inflorescence more or less erect. Petiole 2-48 mm long. - Peninsular Malaysia $\ldots \ldots \ldots \ldots \ldots \ldots \ldots \ldots \ldots \ldots \ldots \ldots \ldots \ldots \ldots \ldots \ldots \ldots \ldots \ldots \ldots$ a. subsp. pubescens

b. Inflorescence more or less pendulous. Petiole 3-20 mm long. - Palawan Island b. subsp. elmerianum

a. subsp. pubescens - Fig. 6; Plate 5a, b; Map 13

Plants $35-150 \mathrm{~cm}$ tall. Petiole of cauline leaves $2-48 \mathrm{~mm}$ long; lamina 30-140 by 14-46 mm. Inflorescences \pm erect; bracts $3-9.7$ by $1.7-4 \mathrm{~mm}$.

Distribution - Thailand and West Malaysia.

Ecology - Flowering: November to April.

Note - The morphological distinction between the two subspecies of Microrphium pubescens is rather insignificant. According to Regalado \& Soejarto (1997), subsp. elmerianum in contrast to subsp. pubescens has yellowish brown pubescent sessile

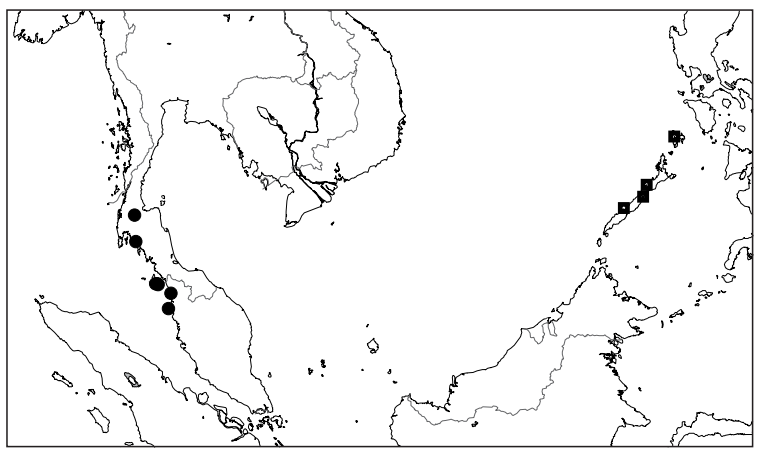

Map 13. Distribution of Microrphium pubescens C.B. Clarke subsp. pubescens $(\bigcirc)$ and M.pubescens subsp. elmerianum (Regalado \& Soejarto) Thiv (ם). 


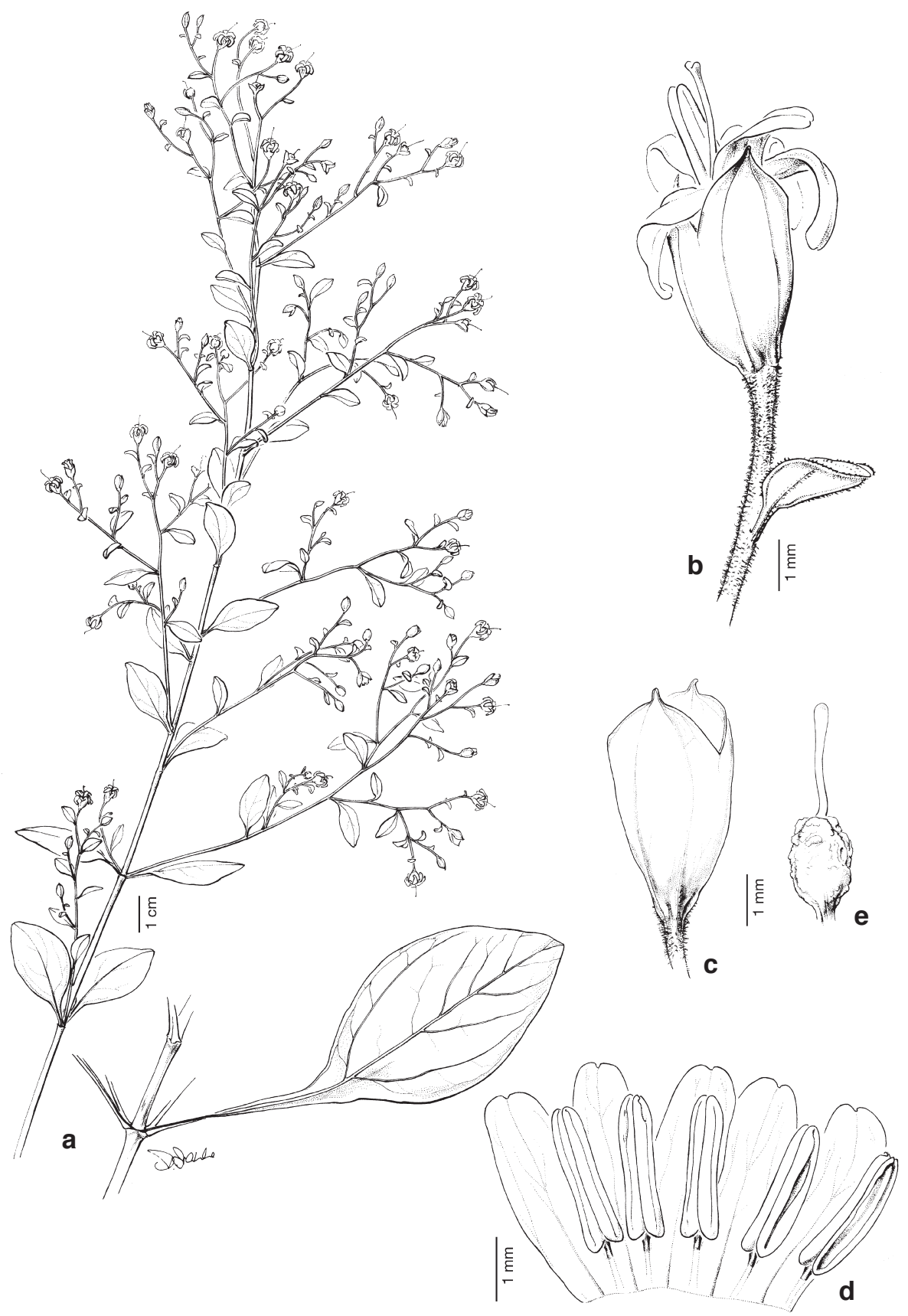

Fig. 6. Microrphium pubescens C.B. Clarke subsp. pubescens. a. Habit; b. flower; c. calyx; d. corolla with stamens; e. gynoecium (a, b: Robinson 6184; c-e: Henderson 29184). 
leaves and glabrous inflorescences. However, the range of leaf size of both subspecies overlaps and differences in the indumentum could not be confirmed. Still, subsp.elmerianum is distinguished by its pendulous inflorescences and somewhat shorter petioles. This together with their geographical distinction is the basis for their recognition as subspecies (cf. Du Rietz, 1930).

b. subsp. elmerianum (Regalado \& Soejarto) Thiv, comb. \& stat. nov. - Map 13

Microrphium elmerianum Regalado \& Soejarto (1997) 77. - Type: Soejarto et al. 6499 (holo F; iso A n.v., PHN n.v.), Philippines, Palawan Island, Puerto Princesa, Bacungan hills, slopes above Nagtabon beach, $9^{\circ} 51^{\prime} \mathrm{N}, 118^{\circ} 37^{\prime} \mathrm{E}, 50-100 \mathrm{~m}, 9$ April 1989.

Plants 35-110 cm tall. Petiole of cauline leaves 3-20 mm long; lamina 35-80 by $16-30 \mathrm{~mm}$. Inflorescences mostly \pm pendulous; bracts $3-6$ by $1.2-3 \mathrm{~mm}$.

Distribution - Palawan.

Ecology - Flowering: February to August.

\section{PHYLLOCYCLUS Kurz - Fig. 7}

Phyllocyclus Kurz (1873) 235. - Type: Phyllocyclus helferianus Kurz.

Canscora Lam. (1785) 601 p.p.

Euphorbiopsis H. Lév. (1911) 446. - Type: Euphorbiopsis lucidissima (H. Lév. \& Vaniot) H. Lév.

Annual or perennial, erect, glabrous herbs; stems apically branched, terete usually without ridges. Rosette or most basal cauline leaves present, usually free, ovate to broadly ovate; cauline leaves perfoliate, orbicular, with several main veins, apex obtuse. Inflorescences axillary, few-flowered, lax cymes; bracts perfoliate, orbicular or funnelshaped. Flowers (except calyx) pentamerous, mostly sessile, rarely pedicellate, without bracteoles. Calyx penta- or tetramerous, inflated urceolate or tubular, without wings, persistent. Corolla actinomorphic, urn-, funnel- to salvershaped, white-yellow to cream-coloured; tube longer than lobes. Androecium isomorphic or anisomorphic with filaments of different length; stamens inserted equally in central or upper part of

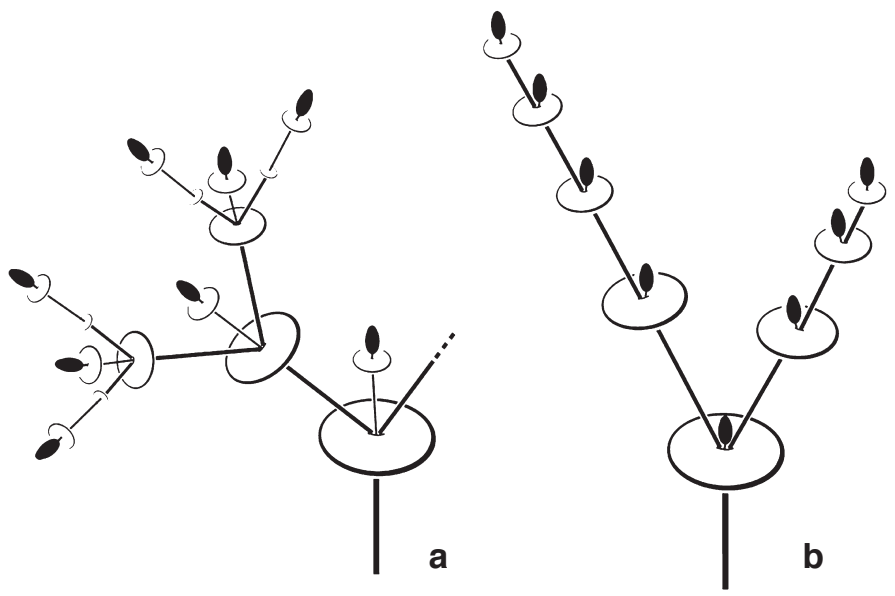

Fig. 7. a. Dichasial and b. monochasial inflorescences of Phyllocyclus. 
corolla tube; filaments broadened at the base; anthers sagittate, deciduous. Ovary ovate to obovoid; surface smooth or constricted; stigmatic lobes rounded. Seeds irregular in shape, subglobose to elliptic; testa cells irregular in shape, oriented irregularly; anticlinal walls undulating, multiply folded; cuticle with scaly exudates.

Distribution - Five species from Burma to southern China.

Notes - With its equal stamen insertion, its mostly pentamerous corollas, and its perfoliate, orbicular cauline leaves, this genus is clearly distinct from Canscora s.str. By sharing these characters Canscora lucidissima and C.petelotii have been transferred to Phyllocyclus.

Unfortunately, species of Phyllocyclus seem to be extremely rare and have been collected only a few times. Therefore, the full range of variation in these species is yet to be determined.

\section{KEY TO THE SPECIES OF PHYLLOCYCLUS}

1a. Flowers mostly in monochasia. Flowers without minute prophylls (Fig. 7) ... . 2

b. Flowers mostly in dichasia. Some flowers with minute perfoliate prophylls (Fig. 7)

2a. Filaments of \pm equal length. Corolla $<9 \mathrm{~mm}$ long $\ldots \ldots \ldots$ 6.1. P. helferianus

b. Some filaments up to 3 times longer than others within one flower. Corolla $>14$

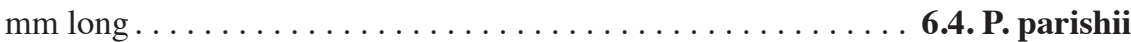

3a. Filaments of \pm equal length. Plants probably perennial $\ldots$ 6.2. P. lucidissimus

b. Some filaments up to 3 times longer than others within one flower. Plants annual

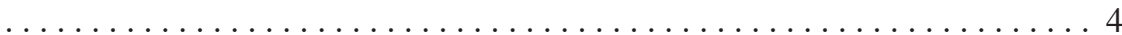

4a. Corolla $<10 \mathrm{~mm}$ long. Bracts funnelform. Calyx urceolate . 6.3. P. minutiflorus b. Corolla $>14 \mathrm{~mm}$ long. Bracts orbicular. Calyx tubular ....... 6.5. P. petelotii

\subsection{Phyllocyclus helferianus Kurz - Plate 5g, h; Map 14}

Phyllocyclus helferianus Kurz (1873) 235. - Canscora helferiana (Kurz) C.B. Clarke (1875) 432. - Type: Helfer 5816 (lecto K, designated here; iso A), Tennasserim and Andamans, Three Pagoda Pass, 1837.

Annual herbs $9-47 \mathrm{~cm}$ tall. Rosette leaves broadly ovate, 18 by $9 \mathrm{~mm}$, early deciduous, apex cuspidate; cauline leaves $9-22$ by $18-27 \mathrm{~mm}$. Inflorescences axillary, 1-15-flowered, lax cymes (mostly monochasia); bracts orbicular $5-10$ by $9-18 \mathrm{~mm}$, without small prophylls (Fig. 7). Flowers (except calyx) pentamerous, (rarely tetramerous), sessile. Calyx tetramerous, inflated urceolate, $7-8$ by $4-5 \mathrm{~mm}$; tube $5.5-7 \mathrm{~mm}$ long; lobes triangular, 1 by $1.3 \mathrm{~mm}$, apex acute. Corolla urn- to salvershaped, 7-9 mm long, creamcoloured to yellow-white; lobes slightly spathulate, apex obtuse, $1-2.3$ by $0.8-1.1 \mathrm{~mm}$. Androecium isomorphic, filaments of equal length, 2.2-2.3 mm long; anthers 1 by 0.4 mm. Ovary ovate with an apical annulus, surface constricted, $2.9-4.5$ by $1.1-3 \mathrm{~mm}$; style up to $5.5 \mathrm{~mm}$ long; stigmatic lobes rounded, 0.3 by $0.4 \mathrm{~mm}$. Capsule oblong, 4 by $3 \mathrm{~mm}$. Seeds c. 0.23 by $0.18 \mathrm{~mm}$.

Distribution - Thailand and Burma.

Ecology - Wet evergreen forest; on calcareous rocks or wet ground. Altitude 100-1200 m. Flowering: September to February. 


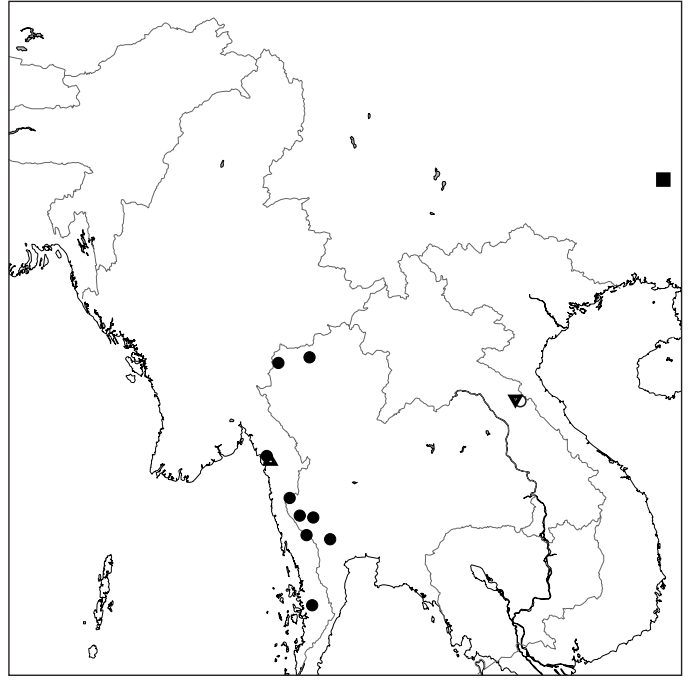

Map 14. Distribution of Phyllocyclus helferianus Kurz (๑), P. lucidissimus (H. Lév. \& Vaniot) Thiv (ם), P. minutiflorus Thiv (O), P. parishii (Hook.f.)

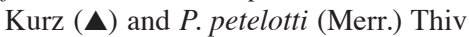
$(\boldsymbol{\nabla})$.

6.2. Phyllocyclus lucidissimus (H. Lév. \& Vaniot) Thiv, comb. nov. - Plate 6c, d; Map 14

Euphorbia lucidissima H. Lév. \& Vaniot (1906) 763. - Euphorbiopsis lucidissima (H. Lév. \& Vaniot) H. Lév. (1911) 446. - Canscora lucidissima (H.Lév. \& Vaniot) Hand.-Mazz. (1931) 234. - Type: Léveillé 1881 (holo E), Sud de Lin Fan, route des rocher.

Apparently perennial herbs $10-30 \mathrm{~cm}$ tall. Rosette leaves ovate, 1.5 by $0.7 \mathrm{~mm}$, early deciduous, apex acute; cauline leaves 11-16 by 21-24 mm. Inflorescences axillary, 3-12-flowered, lax cymes (mostly dichasia); bracts orbicular, $5-8$ by $10-13 \mathrm{~mm}$, with small prophylls (Fig. 7). Flowers (except calyx) pentamerous, sessile. Calyx tetra- or pentamerous, inflated urceolate, $4.7-6$ by $2.8 \mathrm{~mm}$; tube $4 \mathrm{~mm}$ long; lobes triangular, 1.5 by $1.5 \mathrm{~mm}$, apex acute. Corolla urn- to salvershaped, $6-8 \mathrm{~mm}$ long, white or pale yellow; lobes slightly spathulate, $1.9-2$ by $1 \mathrm{~mm}$, apex obtuse. Androecium isomorphic, filaments of equal length, $2-2.3 \mathrm{~mm}$ long; anthers $0.7-1$ by $0.4 \mathrm{~mm}$. Ovary ovate, surface constricted, $3.5-5$ by $1.8 \mathrm{~mm}$; style up to $3.5 \mathrm{~mm}$ long; stigmatic lobes rounded, 0.3 by $0.3 \mathrm{~mm}$; Capsule ovate to oblong, 3.5 by $2.5 \mathrm{~mm}$. Seeds c. 0.19 by $0.13 \mathrm{~mm}$.

Distribution - China.

Ecology - Limestone rocks and slopes. Altitude 1000-1200 m. Flowering: March, August.

\subsection{Phyllocyclus minutiflorus Thiv, spec. nov. - Map 14}

Diagnosis: Species nova $P$. petelotii (Merr.) Thiv similis sed differt corolla parviore et bracteis infundibularibus. - Typus: Pételot 3857 (holo NY), Si Cammon, Village de Cham, sol très humide d'une grotte sur un rocher calcaire, 160 m, Dec. 1930.

Probably annual herbs $12-23 \mathrm{~cm}$ tall. Basal cauline leaves ovate to broadly ovate, $35-37$ by $24-30$, petiolate, petiole $4-10 \mathrm{~mm}$ long, apex obtuse; cauline leaves $7-10$ by 14-23 mm. Inflorescences 3-8-flowered, lax cymes (mostly dichasia); bracts fun- 
nelshaped, $5-6$ by $7-11 \mathrm{~mm}$, with small prophylls (Fig. 7). Flowers (except calyx) pentamerous, pedicellate, pedicels up to $0.5 \mathrm{~mm}$ long. Calyx tetra- or pentamerous, tubular to urceolate, 6 by $2.2-2.8 \mathrm{~mm}$; tube $4.5 \mathrm{~mm}$ long; lobes triangular to needleshaped, 1.5 by $1 \mathrm{~mm}$, apex acuminate. Corolla funnel- to salvershaped, $7 \mathrm{~mm}$ long, probably white or pale yellow; lobes wedgeshaped to broadly spathulate, 2 by $1.8-2$ $\mathrm{mm}$, apex obtuse. Androecium anisomorphic with filaments of different length, 1-3 $\mathrm{mm}$ long; anthers 0.8 by $0.3 \mathrm{~mm}$. Ovary elliptic to ovate, surface smooth, 3 by 2.2 $\mathrm{mm}$; style up to $4 \mathrm{~mm}$ long; stigmatic lobes rounded, 0.3 by $0.3 \mathrm{~mm}$. Capsule oblong, 4 by $2.5 \mathrm{~mm}$.

Distribution - This species is only known from two collections in Laos.

Ecology - Calcareous rocks. Flowering: December.

Etymology - The name refers to the smaller flowers of $P$. minutiflorus compared with the remainder of this genus.

Note - With its filaments of differing length, P. minutiflorus is closely related to $P$. petelotii, but differs from this and other species by having much smaller flowers and funnelshaped bracts.

\subsection{Phyllocyclus parishii (Hook.f.) Kurz - Plate 6a, b; Map 14}

Phyllocyclus parishii (Hook.f.) Kurz (1873) 235. - Canscora parishii Hook.f. (1864) t. 5429. - Type: Parish 451 (A, E, K), Moulmein, limestone rocks, 1862.
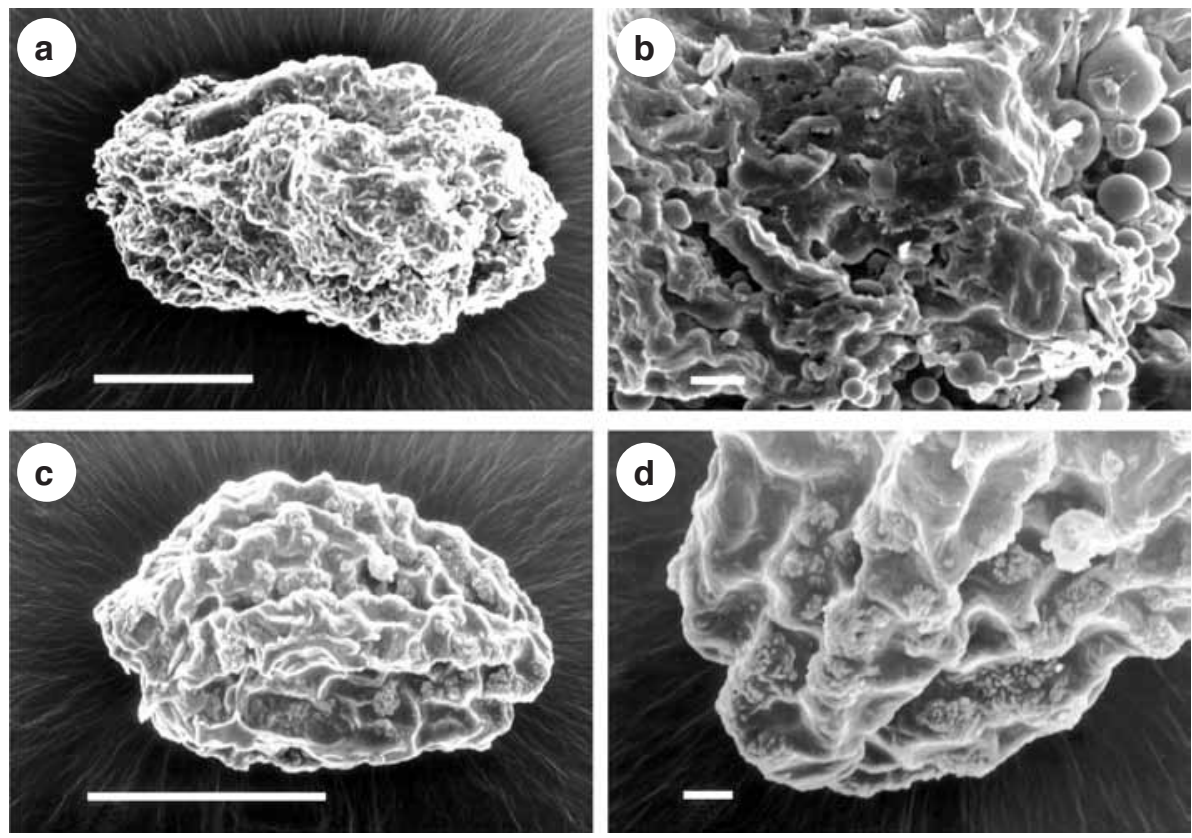

Plate 6. SEM photographs of seed coat structures. - a, b. Phyllocyclus parishii (Hook.f.) Kurz (Gower s.n.); c, d. Phyllocyclus lucidissimus (H. Lév. \& Vaniot) Thiv (Huang s.n.). - Scale bars: $\mathrm{a}, \mathrm{c}=100 \mu \mathrm{m} ; \mathrm{b}, \mathrm{d}=10 \mu \mathrm{m}$. 
Annual herbs 20-40 cm tall. Basal cauline leaves free or perfoliate, early deciduous; cauline leaves $16-38$ by 26-60 mm. Inflorescences mostly axillary, 4-10-flowered, lax cymes (mostly monochasia); bracts orbicular, $6-12$ by $10-25 \mathrm{~mm}$, without small prophylls (Fig. 7). Flowers pentamerous, pedicellate; pedicels up to $2 \mathrm{~mm}$ long. $\mathrm{Ca}$ lyx inflated urceolate, $9-11$ by $4-5 \mathrm{~mm}$; tube $6-8 \mathrm{~mm}$ long; lobes triangular, 3 by 2 $\mathrm{mm}$, apex acute. Corolla \pm salvershaped, 14-19 mm long, white or pale yellow; lobes spathulate, 4-6 by 4-7 mm, apex obtuse. Androecium anisomorphic with filaments of different length, 2-7 mm long; anthers 3 by $0.6 \mathrm{~mm}$. Ovary oblong-ovate with an apical annulus, surface striate, 4.5 by $2 \mathrm{~mm}$; style up to $7 \mathrm{~mm}$ long; stigmatic lobes rounded, 0.8 by $0.6 \mathrm{~mm}$. Capsule oblong, 6 by $3 \mathrm{~mm}$. Seeds c. 0.29 by $0.16 \mathrm{~mm}$.

Distribution - Burma.

Ecology - Limestone rocks.

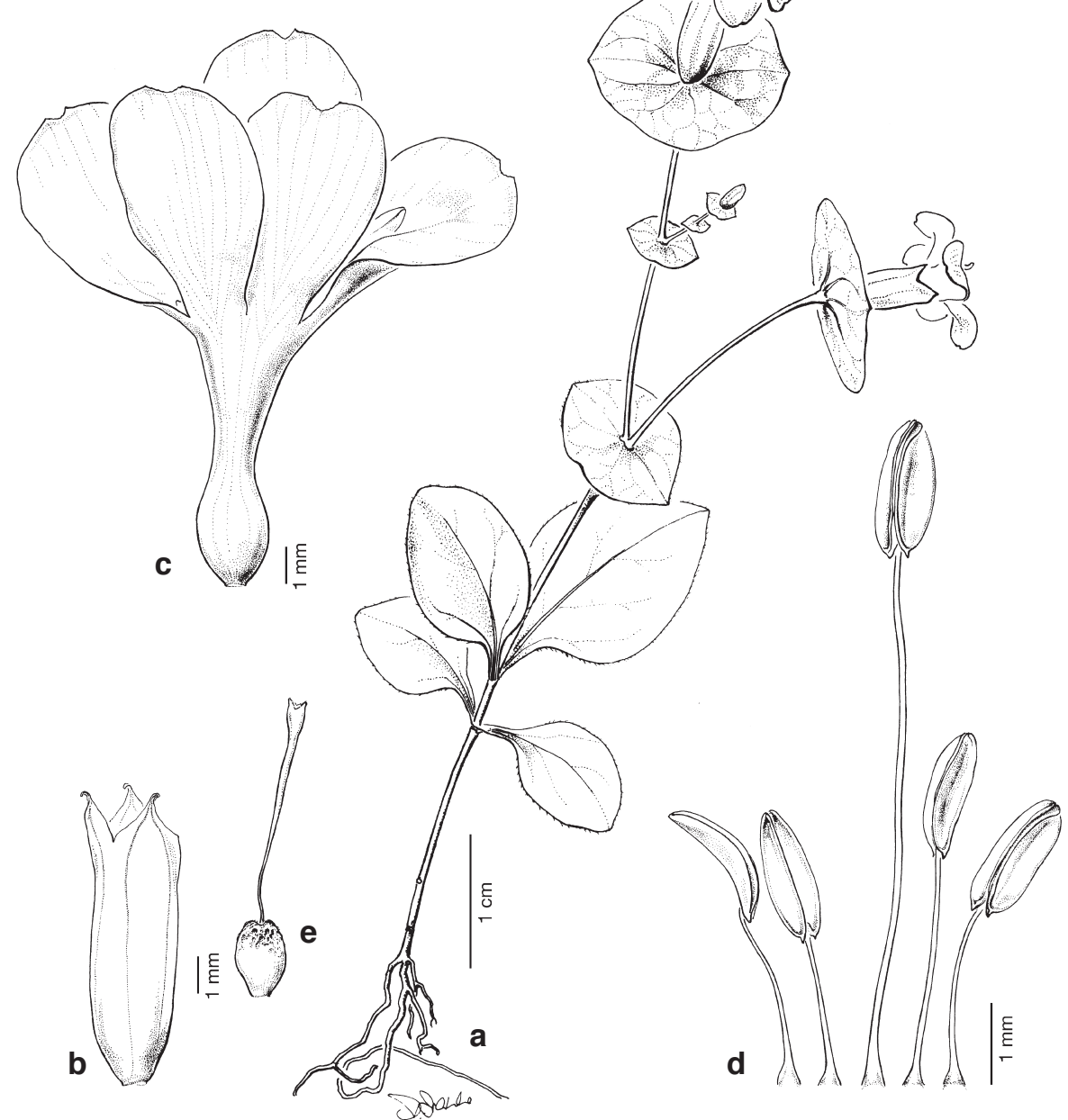

Fig. 8. Phyllocyclus petelotii (Merr.) Thiv. a. Habit; b. calyx; c. corolla; d. stamens; e. gynoecium (all: Pételot 4327). 


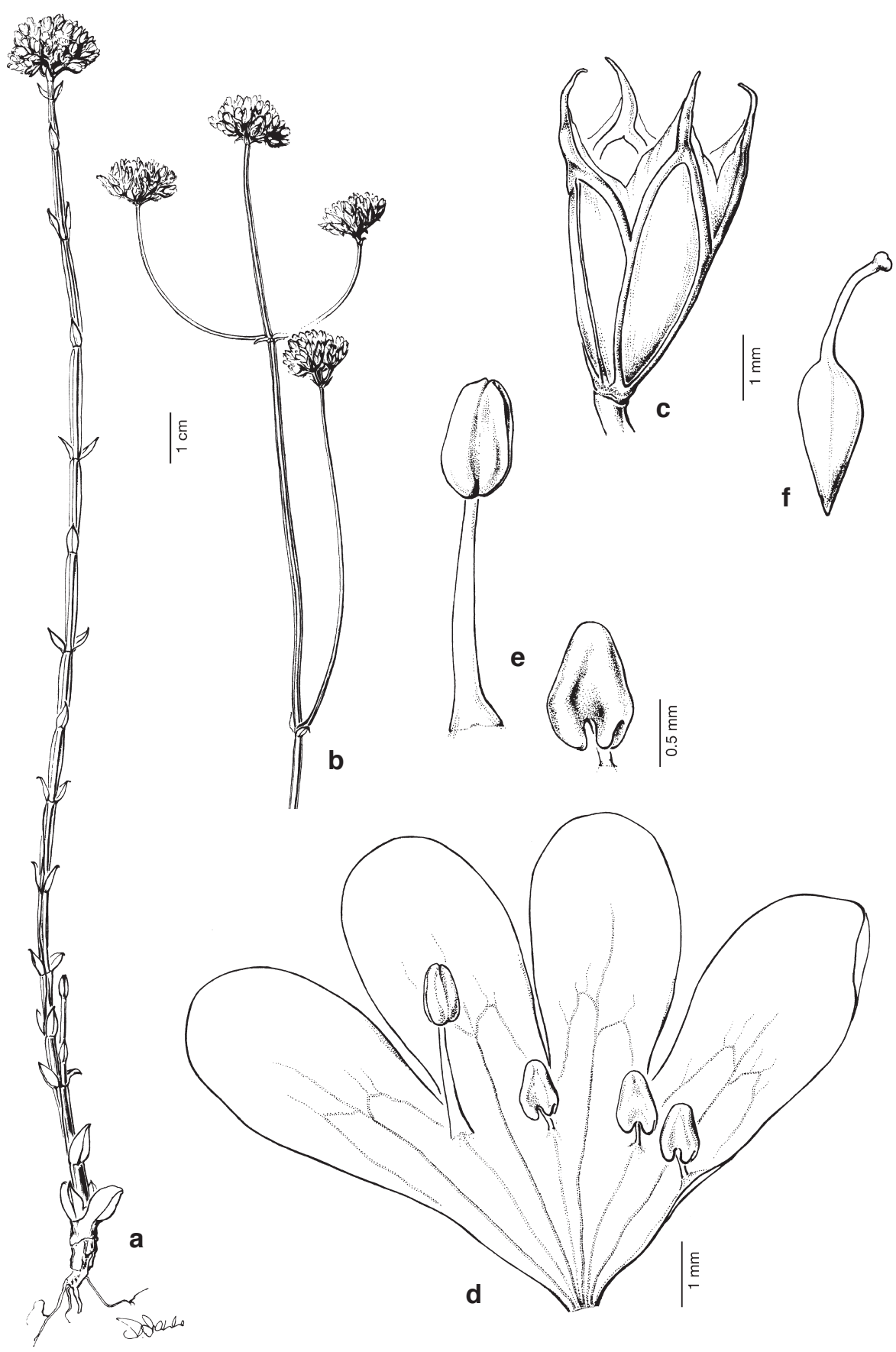

Fig. 9. Schinziella tetragona (Schinz) Gilg. a. Habit; b. inflorescence; c. calyx; d. corolla with stamens; e. stamens of anisomorphic androecium; f. gynoecium (a: Richards 4721; b: Champluvier 5136; c-f: Milne-Redhead 4394). 
6.5. Phyllocyclus petelotii (Merr.) Thiv, comb. nov. - Fig. 8; Map 14

Canscora petelotii Merr. (1938) 63. - Type: Pételot 4327 (holo P n.v.; iso NY), Laos, Si Cammon, Village de Kouan Pha Vang.

Annual herbs $5-9 \mathrm{~cm}$ tall. Basal cauline leaves ovate, 19-20 by 13-18, apex acute; cauline leaves $6-13$ by $8-32 \mathrm{~mm}$ obtuse. Inflorescences few-flowered, lax cymes (mostly dichasia); bracts orbicular, 7-8 by $11-17 \mathrm{~mm}$, with small prophylls (Fig. 7). Flowers (except calyx) pentamerous, pedicellate; pedicels up to $2 \mathrm{~mm}$ long. Calyx trimerous, tubular, $6-7$ by $2.2 \mathrm{~mm}$; tube $5-6 \mathrm{~mm}$ long; lobes triangular, 1.5 by $1.8 \mathrm{~mm}$, apex acute. Corolla funnel- to salvershaped, 14-15 mm long, white or pale yellow; lobes ovate to spathulate, 7 by $4-4.5 \mathrm{~mm}$, apex obtuse. Androecium anisomorphic with filaments of different length, $1.8-6.2 \mathrm{~mm}$ long; anthers 1.7 by $0.8 \mathrm{~mm}$. Ovary obovoid, surface smooth, 2.5 by $1.5 \mathrm{~mm}$; style up to $4 \mathrm{~mm}$ long; stigmatic lobes rounded, 0.3 by $0.3 \mathrm{~mm}$. Capsule oblong, 4.5 by $3 \mathrm{~mm}$.

Distribution - Laos.

Ecology - Calcareous rocks. Flowering: December.

\section{SCHINZIELLA Gilg}

Schinziella Gilg (1895) 74. - Type: Schinziella tetragona (Schinz) Gilg.

Canscora Lam. (1785) 601 p.p.

Perennial, erect, glabrous herbs; stems basally and apically sparsely branched, quadrangular, strongly winged. Basal cauline leaves free, sessile, deciduous, lamina with one main vein, elliptic, base shortly attenuate, apex obtuse or acute; upper cauline leaves free, sessile, lamina with one main vein, broadly lanceolate to triangular, apex acute. Inflorescences axillary and terminally, many-flowered, dense, headlike cymes; bracts free. Flowers tetramerous, pedicellate, sometimes with two long lanceolate bracteoles. Calyx funnelshaped, with ridges at the margin of each sepal, persistent; lobes triangular to blunt with a point. Corolla actinomorphic, funnelform, mostly yellowish creamish; tube \pm as long as the lobes. Androecium anisomorphic (Fig. 9d), stamens inserted in upper or lower part of corolla tube; one or rarely two upper stamens with longer filament and larger anther than the remaining lower stamens; anthers sagittate, persistent. Ovary oblong to elliptic; stigmatic lobes rounded to oblong. Fruit an oblong capsule. Seeds irregular in shape, angular, often cubical to rectangular and with shallowly sunken sides; outer testa reticulate, with shallow cells; testa cells irregularly polygonal, oriented irregularly; anticlinal walls prominent, mostly straight; cuticle smooth.

Distribution - One species in tropical Africa.

\subsection{Schinziella tetragona (Schinz) Gilg — Fig. 9; Plate 4a, b; Map 15}

Schinziella tetragona (Schinz) Gilg (1895) 74. - Canscora tetragona Schinz (1891) 338. - Type: Mechow 418 (lecto Z, designated here; iso BR, G), West Afrika, Malange, 17 Feb. 1891.

Plants 21-62 cm tall with woody rhizome; stems with wings up to $2 \mathrm{~mm}$ wide. Basal cauline leaves $8.5-22$ by $5-17 \mathrm{~mm}$; upper cauline leaves $4-8.9$ by $0.9-3.6 \mathrm{~mm}$. Inflorescences $20-40$-flowered dense cymes; bracts lanceolate, $2.8-8$ by $0.3-2.5 \mathrm{~mm}$; pedicels $0-3 \mathrm{~mm}$ long. Calyx $5-7$ by $2.5-3 \mathrm{~mm}$; tube $2.9-4.5 \mathrm{~mm}$ long; lobes $2-3$ 


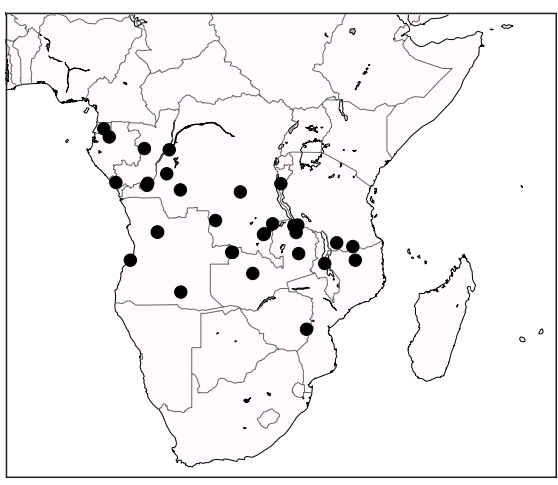

Map 15. Distribution of Schinziella tetragona (Schinz) Gilg.

by $1.5 \mathrm{~mm}$ long. Corolla $6-9 \mathrm{~mm}$ long; lobes obovate to broadly elliptic, $3.5-5.8$ by $1.8-2.3 \mathrm{~mm}$. Filament of the upper stamen $0.9-1.9 \mathrm{~mm}$ long, anther $0.8-0.9$ by $0.4-0.7$ $\mathrm{mm}$; filaments of the lower stamens $0.3-0.4 \mathrm{~mm}$ long, anthers $0.8-1$ by $0.4-0.7 \mathrm{~mm}$. Ovary $2.5-3.4$ by $1.1-1.4 \mathrm{~mm}$; style $1-2.7 \mathrm{~mm}$ long; stigmatic lobes 0.4 by $0.3 \mathrm{~mm}$. Fruit $3-3.5$ by $1.8-2 \mathrm{~mm}$. Seeds $0.23-0.34$ by $0.14-0.21 \mathrm{~mm}$.

Distribution - Tropical Africa.

Ecology - Wet places in sometimes flooded grasslands, swamps and marshes; sandy soil. Altitude 400-1900 m. Flowering: throughout the year.

Note - Schinziella tetragona is variable in its flower size.

\section{ACKNOWLEDGEMENTS}

I would like to thank Joachim W. Kadereit (Mainz, Germany) and an anonymous reviewer for precious and helpful comments on the manuscript. I owe thanks to Doris Franke (Mainz, Germany) for preparing the figures and plates and to Peter Leins and Birgit Volz (Heidelberg, Germany) for accession and support to SEM analyses. I am grateful to Werner Greuter (Berlin, Germany) who helped clarify some nomenclatural problems and to Ferry Bouman (Amsterdam, The Netherlands) who reviewed the seed descriptions. Finally, I thank the directors of A, B, BKF, BM, BO, BR, C, E, F, FWM, G, HBG, K, KLU, KUN, L, MEL, MICH, MO, NSW, NY, P, PE, PH, PNH, S, SING, SRGH, TUB, U, US and $\mathrm{Z}$ for the loan of their material.

\section{REFERENCES}

Bentham, G. \& J. Hooker. 1876. Gentianeae. Genera plantarum, Vol. 2: 799-820. Reeve, London. Berhaut, J. 1975. Flore illustrée du Sénégal. Vol. 4. Claireafrique, Dakar.

Brown, N.E. 1903. Canscora kirkii. In: W.T. Thiselton-Dyer, Flora of tropical Africa. Vol. 4, 1: 588. Reeve, London.

Brown, R. 1810. Prodromus Florae Novae-Hollandiae et insulae Van Diemen. Vol. 1. Johnson, London.

Chen, X.X. 1986. A new species of Canscora from Guangxi. Huihaia 6: 177-178.

Christopher, J. 1976. IOPB-chromosome number report 52. Taxon 25: 344.

Clarke, C.B. 1875. Notes on Indian Gentianaceae. J. Linn. Soc., Bot. 14: 423-457.

Clarke, C.B. 1885. Gentianaceae. In: J.D. Hooker (ed.), The Flora of British India. Vol. 4: 93-132. Reeve, London.

Clarke, C.B. 1906. Flora of the Malayan Peninsula - Gentianaceae. J. Asiat. Soc. Bengal 74: $86-91$.

Dalzell, N.A. 1850. Contributions to the botany of Western India. J. Kew Gard. Misc. 2: 133-145. 
Don, G. 1838. A general history of the dichlamydeous plants. Vol. 4. Corolliflorae. Rivington, London.

Dop, P. 1912. Gentianaceés nouvelles de l'Indo-Chine. Bull. Soc. Bot. France 59: 145-147.

Du Rietz, G.E. 1930. The fundamental units of biological taxonomy. Svensk Bot. Tidskr. 24: $333-428$.

Endlicher, S.L. 1838. Genera plantarum secundum ordines naturales disposita. Beck, Vienna.

Gagnepain, F. 1929. Un genre nouveau de Gentianacées. Bull. Soc. Bot. France 76: 776-777.

Gilg, E. 1895. Gentianaceae. In: A. Engler \& K. Prantl (eds.), Die natürlichen Pflanzenfamilien 4, 2: 50-108. Engelmann, Leipzig.

Gilg-Benedict, C. 1939. Beiträge zur Morphologie und Systematik der Gentianoideae-GentianeaeErythraeinae. Notizbl. Bot. Gart. Berlin-Dahlem 125: 417-430.

Gmelin, J.F. 1791. Systema naturae. Vol. 2. Beer, Leipzig.

Grisebach, A.H.R. 1839. Genera et species Gentianearum. Cotta, Stuttgart, Tübingen.

Grisebach, A.H.R. 1845. Gentianaceae. In: A. de Candolle (ed.), Prodromus systematis naturalis regni vegetabilis. Vol. 9: 39-141. Treuttel, Würtz, Paris, London, Straßburg.

Handel-Mazzetti, H. 1931. Symbolae Sinicae. Vol. 7. Springer, Wien.

Handel-Mazzetti, H. 1932. Plantae novae chingianae. Sinensia 2: 1-7.

Ho, T.N. \& J.S. Pringle. 1995. Gentianaceae. In: Z.-Y. Wu \& P.H. Raven (eds.), Flora of China. Vol. 16: 1-139. Science Press, Beijing, Missouri Botanical Garden, St. Louis.

Hooker, J.D. 1864. Curtis's Botanical Magazine. Vol. 20, Reeve, London.

Hul, S. In press. Gentianaceae. In: S. Hul (ed.), Flore du Cambodge, du Laos et du Vietnam. Muséum National d'Histoire Naturelle, Paris.

Kerr, A.F.G. 1940. Contributions to the Flora of Siam (Thailand). Kew Bull. (1940): 180-186.

Klackenberg, J. 1990. Gentianaceae. In: P. Morat, Flore de Madagascar et des Comores: 5-167. Museum National d'Histoire Naturelle, Paris.

Knoblauch,E. 1894. Beiträge zur Kenntnis der Gentianaceae. Bot. Centralbl. 60: 321-334,353-363, $385-401$.

Kshetrapal, S. 1973. Vascular anatomy of the node and flower of Hoppea dichotoma Willd. Bot. Gaz. (London) 134, 1: 1-4.

Kurz, W.S. 1873. New Burmese plants III. J. Asiat. Soc. Bengal 42: 227-254.

Lamarck, J.B.A.P. de Monnet de. 1785. Canscora. Encyclopédie méthodique. Botanique 1: 601. Panckoucke, Paris.

Léveillé, H. 1911. Decades plantarum novarum LIX-LXX. Feddes Repert. 9: 441-463.

Léveillé, H. \& E. Vaniot. 1906. Les Euphorbia chinois. Bull. Herb. Boissier, sér. 2, 6: 759-764.

Linné, C. 1767. Mantissa Plantarum. Stockholm.

Mallikarjuna, M.B., A. Sheriff \& D.G. Krishnappa. 1989. Chromosome Number Reports 97. Taxon 36: 766-767.

Merrill, E.D. 1938. New or noteworthy Indo-Chinese plants. J. Arnold Arbor. 19: 21-70.

Miquel, F.A.W. 1852. Gentianeae. Anal. Bot. Ind. 3: 10-11.

Miquel, F.A.W. 1856. Flora van Nederlandsch-Indië. Vol. 2. Fleischer, Leipzig.

Naik, V.N. \& D.S. Pokle. 1985. Novelties in the Flora of Marathwada. J. Econ. Taxon. Bot. 7: 670-675.

Paul, S.R. 1977. A new forma of Hoppea (Gentianaceae) from India. Acta Bot. Indica 5: 183-184.

Raynal, A. 1967. Le genre Hoppea Willd. (Gentianaceae) en Afrique. Adansonia, sér. 2, 6: 544548.

Regalado, J.C. \& D.D. Soejarto. 1997. The genus Microrphium (Gentianaceae) in the Philippines. Novon 7: 77-80.

Ridley, H.N. 1908. On a collection of plants made by H.C. Robinson and L. Wray from Gunoung Tahan, Pahang. J. Linn. Soc., Bot. 38: 301-336.

Ridley, H.N. 1923. Gentianaceae. In: H.N. Ridley (ed.), Flora of the Malay Peninsula: 432-437. Reeve, London.

Roemer, J.J. \& J.A. Schultes. 1827. Mantissa. Vol. 3. Cotta, Stuttgart.

Roth, A.W. 1818. Exacum alatum. In: J.J. Roemer \& J.A. Schultes (eds.), Systema Vegetabilum. Vol. 3. Cotta, Stuttgart.

Roxburgh, W. 1814. Hortus bengalensis. Mission Press, Serampore. 
Roxburgh, W. 1820. Flora Indica. Vol. 1. Mission Press, Serampore.

Santapau, H. 1949. Novitates Bombayensis. Kew Bull. (1948): 485-492.

Schinz, H. 1891. Zur Kenntnis afrikanischer Gentianaceen. Vierteljahrschr. Naturf. Ges. Zürich 36: 306-339.

Sedgwick, L.J. 1921. New Bombay species. J. Indian Bot. 2: 123-131.

Struwe, L., K. B. von Hagen, J.W. Kadereit, J. Klackenberg, J.S. Nilsson, M. Thiv \& V.A. Albert. 2002. Systematics, character evolution, and biogeography of Gentianaceae, including a new tribal and subtribal classification. In: L. Struwe \& V.A. Albert (eds.), Gentianaceae - Systematics and Natural History: 21-309. Cambridge University Press, Cambridge.

Thiv, M. 2000. Molekulare und morphologische Phylogenie und Biogeographie der GentianaceaeChironieae und eine Revision der Gattungen Canscora, Cracosna, Duplipetala, Hoppea, Microrphium, Phyllocyclus und Schinziella (Gentianaceae-Canscorinae). PhD thesis University of Mainz, Tectum, Marburg.

Thiv, M. \& J.W. Kadereit. 2002. A morphological-cladistic analysis of Gentianaceae-Canscorinae and the evolution of anisomorphic androecia in the subtribe. Syst. Bot. 27: 780-788.

Thiv, M., L. Struwe, V.A. Albert \& J.W. Kadereit. 1999a. The phylogenetic relationships of Saccifolium bandeirae Maguire \& Pires (Gentianaceae) reconsidered. Harvard Pap. Bot. 4: 519-526.

Thiv, M., L. Struwe \& J.W. Kadereit. 1999b. The phylogenetic relationships and evolution of the Canarian laurel forest endemic Ixanthus viscosus (Ait.) Griseb. (Gentianaceae): evidence from matK and ITS sequence variation, and floral morphology and anatomy. Pl. Syst. Evol. 218: 299-317.

Ubolcholaket, A. 1987. Gentianaceae. In: T. Smitinand \& K. Larsen (eds.), Flora of Thailand. Vol. 5, 1: 72-92. Chutima, Bangkok.

Vahl, M. 1794. Symbolae Botanicae. Vol. 3. Möller, Copenhagen.

Van Rheede tot Draakestein, H.A. 1690. Hortus indicus malabaricus. Vol. 10. Commelijn, Amsterdam.

Voigt, J.O. 1845. Hortus suburbanus Calcuttensis. Bishop's College, Calcutta.

Wallich, N. 1831. A numerical list of dried specimens [= Wallich's catalogue]. London.

Wight, R. 1850. Icones Plantarum Indiae Orientalis. Vol. 4. Franck, Madras.

Willdenow, C.L. 1798. Species plantarum. Vol. 1, 2. Nauk, Berlin.

Willdenow, C.L. 1801. Über zehn neue Gattungen von Gewächsen. Ges. Naturf. Freunde Berlin Neue Schriften 3: 433-453.

\section{IDENTIFICATION LIST}

The abbreviations behind the collector numbers refer to the following taxa:

$\begin{aligned} \mathrm{cal} & =\text { Canscora alata } \\ \mathrm{can} & =\text { Canscora andrographioides } \\ \mathrm{cc} & =\text { Canscora concanensis } \\ \mathrm{cd} & =\text { Canscora diffusa } \\ \mathrm{ch} & =\text { Canscora heteroclita } \\ \mathrm{cm} & =\text { Canscora macrocalyx } \\ \mathrm{cp} & =\text { Canscora perfoliata } \\ \mathrm{cr} & =\text { Canscora roxburghii } \\ \mathrm{cs} & =\text { Canscora schultesii } \\ \mathrm{crc} & =\text { Cracosna carinata } \\ \mathrm{crg} & =\text { Cracosna gracilis } \\ \mathrm{cr} & =\text { Cracosna xyridiformis } \\ \mathrm{dh} & =\text { Duplipetala hexagona }\end{aligned}$

$\begin{aligned} \mathrm{dp} & =\text { Duplipetala pentanthera } \\ \mathrm{hd} & =\text { Hoppea dichotoma } \\ \mathrm{hf} & =\text { Hoppea fastigiata } \\ \mathrm{mpe} & =\text { Microrphium pubescens } \\ & \text { subsp. } \text { elmerianum } \\ \mathrm{mpp}= & \text { Microrphium pubescens } \\ & \text { subsp. pubescens } \\ \mathrm{ph} & =\text { Phyllocyclus helferianus } \\ \mathrm{pl} & =\text { Phyllocyclus lucidissimus } \\ \mathrm{pm} & =\text { Phyllocyclus minutiflorus } \\ \mathrm{ppa} & =\text { Phyllocyclus parishii } \\ \mathrm{ppe} & =\text { Phyllocyclus petelotii } \\ \mathrm{st} & =\text { Schinziella tetragona }\end{aligned}$

More information to the collections in Thiv (2000).

Abbayes 882: st - Abbe 10117: dp - Achten 113: st - Ahern 2449: cd; 3382: cd - Aké Assi 6620: cal; 7512: cd; 7554: cd; 14422: cd; 14519: cd - d'Alleizette 68: pl; 492: hd; 1216: cd; 4944: cal - Altmann 538: cd - Anderson 155: cd; 2849: dp - Anglade 1197: ch - Archbold 
1411: cal; 2860: cal - Argent 980: cal - Armit 652: cd - Arnott 2562: cd; 2567: cp; 8564: cal - Arora 6042: cd.

Backer 5015: cd; 20267: cm; 20269: cm; 27062: cm; 27485: cm; 27689: cm; 27734: $\mathrm{cm} ; 27784:$ cm; 29570: cm; 30006: cm; 32657: cd - Bakhuizen van den Brink 960: cd; 3258: cd; 3466: cd - Balansa 1037: can; 1038: can - Balapure 542: cal - Banks \& Solander 1770: cd - Banskuti 265: ch - Barber 5687: cp - Barnes 276: cd; 649: cr; 652: cd; 674: cd; 777: cal; 967: cd; 968: cd; 970: cp; 1658: hf; 1835: cr; TV2: cr - Baron 9976: cd - Barter 806: cd - Baum 642: st - Bavicchi 315: st; 481: cal - Bear 16979: can - Beddome 5360: cd; 5364: ch; 5368: cd; 5369: cd; 5370: cp - Bedi 1517 p.p.: cal - Bell 5730: cp - Benson 341: st - Bequaert 7337: st; 7833: cal - Berhaut 1481: cd; 1860: cal - Best 21244: dp; 21277: dp - Bhattacharyya 12882: cd - Bingham 9408: cd - Blatter 5484: cd - Boivin 2080: cal - Borgesen 202: cp - Bosser 9746: cd; 18009: cal - Bourne 1611: ch; 2176: hd; 2539: cr; 3415: cd; 6289: cd - Bradford C5053: dp - Bremer 1795: can - Breyne 453: st - Bruce 4: cd - Brummit 14066: st; 17001: st; 18369: cd; 18374: cal - Buchanan 25: cd - Bunchuai 1385: ph - Burkill 2256: dp; 2554 : dp; 3361: can - Burtt 6267: st - Buwalda 8041: cd - Byrnes 2603: cd.

Campbell 10: cd; 82: cd - Carlier 349: st - Celestino 4438: cd - Champluvier 5136: st - Chancellor 258: cal - Charoenphol 4229: can - Chattaryie 5736: cal - Chen 7115: can - Cherian 111320: cc - Chermsirivathana 1569: crc - Chevalier 5981: cal - Chin 1728: mpp - Ching 6635: pl; 7866: can; 7868: can - Chun 43898: can - Clarke 6462: can; 7759: cal; 10177: cal; 10358: cd; 14603: can; 16911: cd; 20896: hd; 24685: cd; 24851: cal; 24877: cal; 25020: cd; 31871: hd; 34174: cd; 34622: cd; 37057: cal; 37325: can - Clarkson 6092: cd - Clemens 4229: can - Coert 490: cm; 495: cm; 709: cd; 1144: cd - Conklin 37917: cd - Cook 66: hd; 236: hf; 1029: hf; 5185: hf; 5281: hf - Coombe 36: cd - Cooray 70020226R: ch - Corner 37857: mpp - Coûteaux 1048: st - Cramer 4830: cal; 4883: hf; 4913: cr; 4926: cr; 5047: hf; 5050: ch; 5058: ch; 5075: ch; 5087: ch; 5092: hf; 5099: cr; 5100: cal; 5102: cd; 5104: cr; 5126: cal; 5129: ch; 5143: hf; 5168: cal; 5191: cr - Cuming 1107: cd - Cunningham 83: cd - Curtis 2516: dp; 3779: dp.

Da Graca Epirito Santo 2842: cal - Danser 6457: cd - Davis 69141: dp - Dawe 71: cal - De Giorgi 200: st - De Voogd 764: cm; 2570: cd; 4442: cd; 4685: cd; 14401: cd; 15354: cd; 15394 : cd - De Wilde 2: cal; 4841: cal - Decary 19212: cal; 19213: cal; 19253: cal - Deighton 3307: cd; 4042: cal; 4408: cal; 4462: cal - Deng 7147: can; 7989: can - Devred 17: cd; 132: cal - Ding 1219: cd - Dorelo 200: cm; 351: cm - Drake 558: cal - Drummond 1773: cd; 3966: cal - Dunlop 4577: cd - Duthie 4752: cd; 6427: cd; 8338: cd; 8350: hd; 9572: hd; 22317: cd.

East Hainan Expedition 45: can; 403: can - Edaño 26823: cd - Ekwuno 76975: cal - Elliot 4505: cd - Elmer 8476: cd - Erlanson 5236: cd; 5261: cd - Espirito Santo Exploracoes Botanicas 2365: cd - Esquirol 2650: pl; 3502: cd - Evrard 1911: cal; 6603: st.

Faden 77/81: ch - Fanshawe 4462: cd; 6997: cd; F6652: cd; F6897: cd; F11423: cd - Faulkner 99: cd; 304: cal; 2677: cal; 4118: cal - Fay 5191: cal - Feller B8: cal; B10: cd - Fenix 28252: cd; 30078: cd - Fernandes 444: cd; 529: cd; 556: cd; 605: cd; 660: cd; 906: cd - Fischer 3215: cp - Flamigni 92: cal; 281: cd; 406: cal - Fosberg 35055: cd - Fox 12577: mpp - Frake 36300: cm - Franck 1029: dp - FRI 1632: dp; — Friedberg 418: cd - Fries 178: cd — Friis 531: cal.

Gamble 3260A: cal; 3262: cd; 6737C: cd; 8631: cd; 8706: hd; 8957: cd; 10134: cd; 10882: cd; 13609: hf; 13647: cal; 13755: hd; 13819: cal; 13828: cd; 14070: hd; 14736: cp; 15441: cal; 15630: cp; 18344: cal; 20364: cd; 21140: cal; 21548: ch; 21622: cal; 21701: hd; 23901: cc; 26434: cd - Gan 40036: can - Garrett 1048: cd; 1347: cd - Gbile 63600: cd - Geerling 1575: cal; 1933: cd - Geesink 8081: cd - Geoffroy 461: crx - George 12329: cd - Germain 2306: cd - Gilbert 8401: cd - Gill 240: cd; 253: cal - Gillet 2682: st - Glasgow 9088: dp - Godbole 45014: cd - Gossweiler 3765: st - Govindarajalu HCPM 3009: hf - Greenway 8017: cd; 8371: st; 8585: cd; 13719: cd - Greilinger 1136: cal - Griffith 5815: cal; 5816/1: can - Guangdong 73 Team 2776: can - Gwynne-Vanghar 268: dp.

Haarer 1934: cal - Hain 6498: cd - Hainan Exp. 229: can - Haines 2721: cd - Hallier 4216: cd - Hambler 994: cal - Haniff 4019: mpp; 7076: dp - Hansen 11372: can; 12297: dp; 12355: mpp; 40559: can - Hara 6302627: cal - Hardial 476: dp - Harley 9460: cd - Hartley 13753: 
cd - Haselfoot Haines 4532: hd; 4886: cal - Hauff 7945: can - Helfer 5815: cd; 5816: ph - Helling 245: cm - Hendelot 170: cd - Henderson 19458: dp; 21380: dp; 23118: dp; 29184 : mpp - Henry 12772: can - Hepper 1041: cd; 1387: cal - Heybroek 89e: cd - Hildebrandt 3303: cal - Ho 60019: can - Hohenacker 305: cd; 398: cd; 581: hf; 670: cd; 810: cp - Holstvoogd 16: cd - Holttum 20949: can - Hooper 497: st; 2457: cd; 39312: ch - Horsfield 1279: cm; 3949: cm - Hosseus 287: cd; 387: cd - Hou 713: dp; 73519: can - How 72398: can; 73519: can - Huang 36507: can - Humbert 4942: cd; 18015: cd - Hutchinson 3439: cd.

Inder 93: hd - Irvine 4784: cal - Ismail 26: cd - Iwatsuki 35: cd; 139: cd; 7487: can.

Jaag 1381: cd - Jacobsen 252: cd - Janaki 265: cd; 266: cal; 267: ch; 411: cd; 481: cd - Jans 169: st - Jansen 277: st - Jayaseelan 21921: cal; 21966: hd - Jayasuriya 1137: cr; 2092: hf - Jayaweera 230: cal.

Kanodia 88232: cd; 96314: cd - Kasim 1348: can - Kaul 8878: hd - Keay 25010: cd - Keenan 1932: can; 3044: can; 3833: can — Kelsall 1974: dp - Kenneally 4979: cd - Kern 8274: cd - Kerr 977: cd; 1521: cal; 2298: cal; 2344: cd; 2364: cd; 2378: cd; 2546: cd; 8059: dh; 8226: crx; 9580: crx; 10278: ph; 10531: cd; 10550: ph; 10955: dp; 11329: dp; 11379: dp; 11789: dp; 11875: dp; 14652: dp; 17323: dp; 17641: can; 18079: can; 18455: can; 18926: dp; 21123: can - Khan 74: can; 896: cd; 4748: cd - Kiah 35371: dp - Kiew RK 1526: dp; RK 1617: dp; RK 2987: dp - Kira 99: can - Kirt Ram 8804: cal - Klackenberg 360: cd; 566: cp; 568: cd; 818: dp - Klein 1799: cd - Kloss 12103: can - Koelz 4599: cd; 10770: cd; 13367: cp; 19539: hd; 29638: can - Kolbe 3147: cd - Kooy 325: cd; 937: cd - Kornas 1985: cd - Kororiya 396: cd - Kostermans 1049: cd; 18644: cd - Kotschy 488: cd - Koyama 15530: cd; 15568: cd; 30651: cd; 32404: cd; 32428: cd; 33984: mpp - Kramer 6205: cd - Kuntze 6335: cd; 6357: cal; 7288: cd; 7430: cd; 7431: cd - Kurz 213: cs - Kuswata 155b: cd.

Lace 5478: cal; 6378: cd - Larsen 8591: cd; 9214: ph; 33702: dp; 34343: ph; 42547: dp - Latilo 28758: cal - Lau 2425: can; 3370: can — Laurent 430: cal — Le Testu 1325: cal; 2142: st; 2832: st; 3006: cd; 3366: cal; 3375: cal; 3789: cd; 7766: cal - Leach 11154: cd - Leaño 41653: cd - Leeuwenberg 8995: cd - Lely 703: cd - Léveillé 1881: pl - Lewalle 4294: st - Li 200292: can - Liang 63704: can; 64374: can; 65282: can; 68773: can - Lisowski 12942: st; 21055: cd; 51184: cd - Lobb 345: cd; 350: can; 354: hf; 430: can - Loher 4095: cd; 4096: cd - Lowe 1853: cal - Lowell 3379: cd - Luezon 8296: cd - Luiware 427: cd - Luke 3316: cal.

Madulid 6614: mpe - Maheshwari 4133: hd; 4361: cd - Malaisse 951: cal; 4998: st - Mangubat 379: cd; 979: cd - Masens 1345: st - Matthew 12275: hd; 12283: cd; 16234: ch; 19874: ch; 19943: ch; 20614: cd; 25947: cal; 25951: ch; 28997: ch — Maxwell 78-111: dp; 88-361: cd; 881165: cal; 88-1403: cal; 89-1420: cal; 89-1533: cd; 89-1581: cd; 90-77: cd; 92-69: cd - Mayotte 3215: cal - Mazumdar 10405: cd - McGregor 43632: cd - McKee 5796: cd - McNur 34396: dp - Mearns 2956: cd - Mechow 418: st - Meebold 8471: cp; 10028: cp - Meikle 699: cd - Mendoza 20344: cd - Merrill 92: cd; 115: cd; 155: cd; 216: cd; 651: cd; 15544: cd - Meyer 9642: dp - Micholitz 109: cal - Migeod 558: cd - Military team 236 1511: can - Miller 7571: cc; 9263: cc; M9057A: cc - Milne-Redhead 4394: st; 7422: cd; 7729: st; 8067: st - Miquel 4305: cd; 4398: cd - Missa 908: hd - Mooney 2594: hf; 2612: cd; 2789: hf; 3588: ch; 3601: cal; 3650: ch; 3809: cd - Morat 977: cd - Morton SL 118: cd; SL 673: cd; SL 889: cd; SL 32796: cd - Moyser 31083: can - Mshasha 75: cd - Mt Diaoluo Exp. 2934: can - Mukherjee 4222: cd.

Nai Nol 12691: can - Naithani 3954: cal - Nana 7364: cd - Nangoma 201: cd - Nathan 4361: cd - Nauen 38026: dp - Nautiyal 25375: cd - Ned. Ind. For. Service 6663: cm - Neubauer 5227: cd - Newbould 4299: cal - Nicolson 2276: cd - Niyomdham 160: cd; 436: ph; 1642: dp - Nur 12133: dp.

Olorunfemi 24393: cal - Ooststroom 12605: cd; 12676: cd - Otanes 17840: cd - Overlaet 528: st; 1990: st.

Palmer 149: cd - Panigrahi 4953: can; 6090: cd; 11840: cd - Pant 43243: cd - Parish 451: ppa - Parker 2297: can - Parry 599: can - Pawek 7160: cd; 9386: cal; 11365: cal — Perrier de la Bathie 775: cd; 9062: cd; 9078: cal; 16270: cal - Perrottet 329: cd; 819: cd - Pételot 298: can; 3857: pm; 3857: ppe; 4327: pm - Phengkhlai 695: can; 3491: crc - Phipps 1276: cd; 2172: cd - Pirozynski 682: cal - Polunin 3896: cd; 5779: cal; 5857: cd - Prain 120: can; 174: can; 
571: can - Proshad 30148: cc - Purseglove P1074: cal - Put 1022: dp; 1627: dp; 1925: crc; 2484: dp.

Radcliffe-Smith 5307: cc - Raghavan 79548: cd - Raizada 21358: cd - Rakoto 9548: cd - Rakotovao 4607: cd - Ralph 13: cd; 653: cp - Ramos 4887: cd; 5110: cd; 27429: cd; 27430: hd; 28978: cd; 44683: cd - Rao 71708: cd — Rayado 20567: cd - Reekmans 6939: cal - Reillo 19226: cd - Richards 1440: st; 4721: st; 5211: st - Ridal 31815: cd - Ridley 8214: dp; 8218: dp; 13008: dp; 13400: dp; 16045: can; 16245: can - Ridsdale SMHI 240A: mpe - Risopulos 177: cal - Ritchie 112: cal; 355: cal; 464: cp; 466: cd; 1874: cd - Roberty 6970: cd; 6996: cd; 10238: cd; 16510: cd - Robinson 769: cd; 1652: cd; 3702: cd; 6072: can; 6184: mpp; 6464: st - Robyns 7206: ch; 7206bis: hf - Rodenburg 278: cd - Rogers 5276: cd - Rottler Rounce 396: cal - Rupchand 5274: can; 6794: can; 6862: cd - Rupert 42: hd.

Saldanha 12022: cd; 12115: cp; 12199: cd; 12532: cd; 13354: cp; 15131: hf; 15224: cal; 15508: cd; 15529: cp; 15636: cp; 15757: cd; 15772: cd; 15894: cp; 16463: cp; 56349: cp - Sanane 528: st - Sandwich 177: cd - Santapau 5418: cd; 8765: cd; 11626: cd; 11693: cd; 13373: cd; 13438: cc; 13848: cd; 16158: cd; 17607: cd; 17608: cd; 22116: hd - Santisuk 454: cd; 6853: can - Santos 5886: cd; 6155: cd - Savory 25300: cal - Schaap 83: can - Schiffner 2413: cd; 2417: cd - Schmutz 1535: cm; 2214: cd - Schultz 366: cd - Schweinfurth 130: cd; 2614: cal; 2814 : cd - Scott 18: cd; 420: cd; EAH 11771: cal - Sedgwick 5150: cp; 5381: cd; 5487: cd; 6564: hf; 6595: cd - Seimund 163: can - Seyrig 175: cd - Sharma E 187: cal - Shawe S19: can; S20: can - Shi 12688: cd - Simpson 9628: cal - Sinclair 3262: cd; 3350: cd; 4501: cd; 7826: dp; 9868: dp; 40065: dp - Singh 408: cd - Sinsin 1481: cal; 1482: cd - Smitinand 4182: cd; 7894: can; 7934: can; 8438: crg; 8624: can; 10254: cd; 36363: cd - Soejarto 6362: mpe; 8495: mpe - Soejarto et al. 6499: mpe - Sørensen 964: cd - Spare 36318: dp - Stainton 4160: cal; 7070: cal; 8701: cd-Stone 419: mpe; 5916: dp; 7292: dp; 7460: dp; 8565: can; 8923: dp; 11463 : dp; 14194: mpp - Strachey 1: cd - Strugnell 17071: dp - Subbarao 22264: hd - Subbaras 29674: cal - Sukanagar 395: cal - Supapol 276: dp - Suresh 21783: cd - Swartz 398: cd - Synepton 37433: dp - Synringku 28839: can.

Tagawa 2214: cd - Talbot 2156: cal - Tao 1227: cd - Taylor 3380: cd - Tessier-Yandell 126 C: can - Teijsmann 8739: cd - Thollon 774: cal - Thompson 86: ch - Thor 1615: cal - Thwaites 1874: cr; 2400: cal; 2840: hf; 2841: ch; 3581: ch - Toka 102: st; 128: st - Toppin 4050: can - Townsend 73/5: cd - Tsang 2493: can; 14354: can; 22906: can; 24282: can; 24453: can; 24482: can; 24559: can; 27123: can; 29606: can; 30694: can — Tso 22130: cal.

UNESCO 285: dp.

Van Balgooy 1427: cd; 2217: mpp; 2704: dp - Van Beusekom 22: cd; 68: cd; 1684: can; 2513: cd; 3641: cal; 3814: dh; 3902: 3915: cd; 4369: can; 12698: cd - Van Steenis 12494: cm Vanderyst 1990: st; 3343: st; 3438: st; 5045: cal; 11164: st - Vaughan 102: cal; 632: cal; 2243: cal - Venugopal 22055: cd - Verheijen 2499: cm; 4456: cd - Verschueren 595: cd; 634: cd - Vesterdal 212: can - Vidal 3321: cd.

Walker 7461: cd - Wallace 9108: cd; 9134: hd; 9157: cd - Wallich 4361: can; 4363: cp; 4364: ch; 4365: cr; 4366: hd; 4367: ch; 4368: cs; 4964: cal - Walsch 490: cd - Wang 34696: can; 35865: can; 36507: can; 36572: can; 40010: can; 80636: cal; 80740: cd - Ward 1956: can; 20359: can - Warnecke 298: cal - Waterlot 965: cal - Watt 5063: cd; 5150: can; 6660: can; 7816: cal; 9877: cal - Whight 2565: ch - Whyte 81: cal - Wickens 1526: cd - Wight 1825: hf; 1826: ch; 1828: cr; 1829: cd; 1831: cd; 2562: cd; 2563: cd; 2564: cal; 2566: cp; 2567: cr — Wingfield 4006: cd - Winkler 1802: dp - Wood 75: cd; 180: cal; 235: hd; RHT 30759: hd - Wray 5507: can - Wrigley 137: cal - Wyld 324: cal; 467: cal.

Ye 35636: can - Yü 17673: cd.

Zhang 82: can - Zhong A63490: can - Zollinger 3949: cm; 3979: cd. 


\section{INDEX}

Accepted taxa are in roman type, new taxa in bold and synonyms and insufficiently known (dbt/excl.) in italics. Numbers refer to the species number as used in this revision.

Canscora Lam. [p. 5, 19, 25, 33, 39]

alata (Roth) Wall. 1.1

alata Wall. 1.7

andrographioides Griff. ex C.B. Clarke 1.2

concanensis C.B. Clarke 1.3

decurrens Dalzell 1.4

decussata (Roxb.) Roem. \& Schult. 1.1

diffusa (Vahl) R.Br. ex Roem. \& Schult. 1.4 var. tetraptera Naik \& Pokle 1.4

divaricata Miq. ex C.B. Clarke 1.4

grandiflora Wight 1.7

helferiana (Kurz) C.B. Clarke 6.1

heteroclita (L.) Gilg 1.5

hexagona Kerr 3.1

justicioides Griff. ex Voigt dbt/excl.

khandalensis Santapau 1.4

kirkii N.E. Br. 1.4

lancifolia Miq. ex C.B. Clarke 1.4

lawii Wight 1.4

lucidissima (H. Lév. \& Vaniot) Hand.-Mazz. 6.2

macrocalyx Miq. 1.6

macrocalyx Miq. ex C.B. Clarke 1.7

melastomacea Hand.-Mazz. 1.2

parishii Hook.f. 6.4

pauciflora Dalzell 1.4

pentanthera C.B. Clarke 3.2

perfoliata Lam. 1.7

petelotii Merr. 6.5

roxburghii Arn. ex Miq. 1.8

rubiflora X.X. Chen 1.4

schultesii Wall. ex Griseb. 1.9

sessiliflora (Roxb.) Roem. \& Schult. 1.5

stricta Sedgw. dbt/excl.

tenella Wall. 1.4

tenella Wight 1.4

tetragona Schinz 7.1

trinervia Ridl. 1.2

ventricosa J.F. Gmel. 1.7

wallichii C.B. Clarke 1.8

Canscorinae Thiv \& Kadereit [p. 4]

Cicendia fastigiata Griseb. 4.2
Cracosna Gagnep. [p. 19]

carinata (Dop) Thiv 2.1

gracilis (Dop) Thiv 2.2

xyridiformis Gagnep. 2.3

Duplipetala Thiv [p. 25]

hexagona (Kerr) Thiv 3.1

pentanthera (C.B. Clarke) Thiv 3.2

Euphorbia lucidissima H. Lév. \& Vaniot 6.2

Euphorbiopsis H. Lév. [p. 33] lucidissima (H. Lév. \& Vaniot) H. Lév. 6.2

Exacum alatum Roth 1.1 diffusum (Vahl) Willd. 1.4

heteroclitum (L.) Willd. 1.5

Gentiana diffusa Vahl 1.4

heteroclita L. 1.5

Heterocanscora C.B. Clarke [p. 5] schultesii (Wall. ex Griseb.) C.B. Clarke 1.9

Hoppea Willd. [p. 28] dichotoma Willd. 4.1 forma pedicellata S.R. Paul 4.1 fastigiata (Griseb.) C.B. Clarke 4.2

Microrphium C.B. Clarke [p. 30] elmerianum Regalado \& Soejarto 5.1, 5.1b

pubescens C.B. Clarke 5.1 subsp. elmerianum (Regalado \& Soejarto) Thiv 5.1b

subsp. pubescens $5.1 \mathrm{a}$

Orthostemon R.Br. [p. 5]

erectus R.Br. 1.4

Phyllocyclus Kurz [p. 33]

helferianus Kurz 6.1

lucidissimus (H. Lév. \& Vaniot) Thiv 6.2

minutiflorus Thiv 6.3

parishii (Hook.f.) Kurz 6.4

petelotii (Merr.) Thiv 6.5

Pladera Sol. ex Roxb. [p. 5, 28]

alata Buch.-Ham. ex Wall. 1.7

decussata Roxb. 1.1

fastigiata (Griseb.) C.B. Clarke 4.2

pulchella Wall. 1.9

pusilla Roxb. 4.1

sessiliflora Roxb. 1.5

Schinziella Gilg [p. 39]

tetragona (Schinz) Gilg 7.1 